\title{
A Spatio-Temporal Enhancement Method for medium resolution LAI (STEM-LAI)
}

\author{
Rasmus Houborg $^{1 *}$, Matthew F. McCabe ${ }^{1}$ and Feng Gao ${ }^{2}$ \\ ${ }^{1}$ Water Desalination and Reuse Center, Biological and Environmental Sciences and Engineering \\ Division, King Abdullah University of Science and Technology (KAUST), Saudi Arabia \\ ${ }^{2}$ USDA-ARS, Hydrology and Remote Sensing Laboratory, Beltsville, MD, USA
}

*Corresponding author:

Rasmus Houborg

Hydrology and Land Observation (HALO) group

Biological and Environmental Sciences and Engineering Division

Water Desalination and Reuse Center

King Abdullah University of Science and Technology (KAUST)

Building 4, office 3262

P.O. Box 4090, Thuwal 23955

Kingdom of Saudi Arabia

Cell: +966 544700262

Email: rasmus.houborg@kaust.edu.sa

Keywords: Landsat; MODIS; STARFM; LAI; data fusion, downscaling; multi-scale; regression tree; spatiotemporal enhancement; data mining 


\section{$1 \quad$ Abstract}

Satellite remote sensing has been used successfully to map leaf area index (LAI) across landscapes, but advances are still needed to exploit multi-scale data streams for producing LAI at both high spatial and (STEM-LAI) has been developed to generate 4-day time-series of Landsat-scale LAI from existing medium resolution LAI products. STEM-LAI has been designed to meet the demands of applications requiring frequent and spatially explicit information, such as effectively resolving rapidly evolving vegetation dynamics at sub-field $(30 \mathrm{~m})$ scales. In this study, STEM-LAI is applied to Moderate Resolution Imaging Spectroradiometer (MODIS) based LAI data and utilizes a reference-based regression tree approach for producing MODIS-consistent, but Landsat-based, LAI. The Spatial and Temporal Adaptive Reflectance Fusion Model (STARFM) is used to interpolate the downscaled LAI between Landsat acquisition dates, providing a high spatial and temporal resolution improvement over existing LAI products. STARFM predicts high resolution LAI by blending MODIS and Landsat based information from a common acquisition date, with MODIS data from a prediction date. To demonstrate its capacity to reproduce fine-scale spatial features observed in actual Landsat LAI, the STEM-LAI approach is tested over an agricultural region in Nebraska. The implementation of a $250 \mathrm{~m}$ resolution LAI product, derived from MODIS $1 \mathrm{~km}$ data and using a scale consistent approach based on the Normalized Difference Vegetation Index (NDVI), is found to significantly improve accuracies of spatial pattern prediction, with the coefficient of efficiency $(E)$ ranging from $0.77-0.94$ compared to $0.01-$ 0.85 when using $1 \mathrm{~km} \mathrm{LAI}$ inputs alone. Comparisons against an 11-year record of in-situ measured LAI over maize and soybean highlight the utility of STEM-LAI in reproducing observed LAI dynamics (both characterized by $\mathrm{r}^{2}=0.86$ ) over a range of plant development stages. Overall, STEM-LAI represents an effective downscaling and temporal enhancement mechanism that predicts in-situ measured LAI better than estimates derived through linear interpolation between Landsat acquisitions. This is particularly true when the in-situ measurement date is greater than 10 days from the nearest Landsat acquisition, with prediction errors reduced by up to $50 \%$. With a streamlined and completely automated processing interface, STEM-LAI represents a flexible tool for LAI disaggregation in space and time that is adaptable to different land cover types, landscape heterogeneities, and cloud cover conditions. 


\section{Introduction}

Leaf Area Index (LAI), defined as the one-sided green leaf area per unit horizontal ground area, represents a key remote sensing based descriptor of vegetation density (Gobron et al., 1997). Describing LAI distribution in space and time represents a key input to a range of models, including those used for land surface flux estimation, water resource management and crop growth monitoring (Anderson et al., 2012; Bonan et al., 2011; Doraiswamy et al., 2004; Sellers et al., 1996). In order to effectively resolve vegetation dynamics at the scale of individual fields and to provide meaningful scale-relevant decision support, modeling approaches require inputs of LAI that have both high spatial and high temporal resolution. Time-critical information on within-field variability in crop condition and vegetation biomass is also useful for defining spatially explicit management strategies for optimizing agricultural yield (Moran et al., 1997), and continuous time-series of high resolution LAI can be beneficial for land cover mapping and change detection (Cai et al., 2014) and for land surface phenology characterizations (Walker et al., 2014; Zhang et al., 2006). Unfortunately, resolving the spatial-temporal divide remains a challenge. Indeed, satisfying the simultaneous requirements of timeliness and fine spatial resolution is not currently possible using any single satellite sensor, due to constraints in either temporal or spatial resolution. Whereas medium resolution $(100-1000 \mathrm{~m})$ sensors (e.g., MODIS, MERIS) typically have near-daily revisit times, sensors conveying high resolution (10 - $100 \mathrm{~m}$ ) information (e.g., Landsat, ASTER) most often have inadequate repeat coverage (16 day or more) (Houborg, Fisher, \& Skidmore, 2015). As a result, LAI modeling efforts must rely on multi-sensor approaches in order to accomplish these observational goals.

Satellite remote sensing can be used to infer the spatial and temporal distribution of LAI across a landscape and is one of the most commonly employed metrics with which to monitor vegetation health and function (Anderson et al., 2015; Gitelson et al., 2014). To meet this need, a range of multi-year global datasets of LAI have been made available at resolutions of approximately $1 \mathrm{~km}$, based on medium resolution optical sensors such as MODIS (Knyazikhin et al., 1999) and SPOT/VEGETATION (Baret et al., 2007), or through a combination of sensors (Baret et al., 2013; Pinty et al., 2011; Xiao et al., 2013). While these sensors have near-daily revisit times, the LAI products are composited every 4 to

6116 days in order to increase the availability of high quality cloud free radiometric information as input to 
63 and potentially global LAI from Landsat surface reflectance data, including a physically based algorithm

64 based on canopy spectral invariants theory (Ganguly et al., 2012) and a regression tree methodology that

65 uses high-quality LAI retrievals from MODIS to produce MODIS consistent Landsat-resolution LAI

66 (Gao et al., 2012). However, the 16-day revisit cycle of Landsat, which can be significantly longer due

67 to cloud contamination, limits the use of Landsat as the sole source for reproducing the temporal LAI 68 dynamics that are important for studying rapidly evolving biophysical processes at the land surface (Roy 69 et al., 2008).

71 Gao et al. (2006) developed a data fusion algorithm with the ability to blend aspects of medium and high 72 spatial resolution sensors. The so-called Spatial and Temporal Adaptive Reflectance Fusion Model 73 (STARFM) was designed to capture quantitative changes in surface reflectance and has demonstrated 74 utility in generating maps of surface reflectance that preserve the high spatial resolution of Landsat and 75 the high frequency of MODIS (Emelyanova et al., 2013; Gao et al., 2006; Hilker et al., 2009; Wang et 76 al., 2014). STARFM is extendable to other biophysical properties, so long as the multi-scale input data streams can be corrected to comparable scale-consistent estimates (Gao et al., 2006). An application to multi-scale evapotranspiration (ET) data streams was demonstrated by Cammalleri et al. (2014) facilitating daily mapping of ET at Landsat spatial resolution. More recently, an extension to land surface temperature data has also been proposed (Weng et al., 2014). Successful application of STARFM requires that the sensor-specific data streams are comparable and spatially and temporally consistent, which may involve corrections for differences in orbital characteristics (e.g., overpass time and viewing geometry), spectral bandwidths, atmospheric correction, geolocation accuracy (Wang et al., 2014), and parameter retrieval algorithms. Since STARFM relies on medium resolution information from homogeneous neighbor pixels, prediction accuracies may also degrade when the typical dimension of agricultural field boundaries is less than the scale of the medium resolution data. In these heterogeneous landscapes, the range of conditions evident at the high spatial resolution may not be well represented at the coarser scale (Cammalleri et al., 2014; Hilker et al., 2009; Senf et al., 2015)

The objective of the current study is to develop a flexible application for effective downscaling of

91 relatively high frequency medium resolution LAI products to the high resolution Landsat spatial scale.

92 The Spatio-Temporal Enhancement Method for LAI (STEM-LAI) implements a rule-based regression 93 tree method (Gao et al., 2012) for downscaling medium resolution LAI products during time-coincident 
94 Landsat acquisitions. A refined version of STARFM is applied to effectively interpolate high spatial 95 resolution LAI between Landsat acquisitions. To do this, a number of novel elements were integrated 96 into the STEM-LAI approach, including: 1) the implementation of $250 \mathrm{~m}$ resolution LAI, downscaled

97 from native $1 \mathrm{~km}$ products using a scale-consistent and biome-specific NDVI-based technique; 2) an 98 approach for ensuring consistency between LAI data streams across spatial scales; 3) a sequential 99 image-pair selection strategy for effective gap-filling of areas affected by clouds or scan line corrector 100 off stripes in Landsat imagery; and 4) optimization of STARFM parameterizations and input options for 101 application over a wide range of environmental conditions and Landsat acquisition frequencies. STEM102 LAI is based on image-based methodologies packaged within a fully automated processing 103 infrastructure for seamless generation of temporally continuous (every 4-day) and spatially enhanced (30 $104 \mathrm{~m})$ LAI maps for user-defined regions of interest. It provides a novel interface for exploiting the 105 combined benefits of spatial downscaling and temporal enhancement methodologies in order to meet the 106 demands of applications requiring time-critical information at sub-field scales.

In this study, STEM-LAI is applied to MODIS LAI and Landsat data acquired over a maize and soybean producing region in central Nebraska. The utility of STEM-LAI for predicting spatial and temporal features in actual Landsat LAI images is extensively evaluated for different plant development stages and imposed Landsat acquisition gaps. An 11-year record of weekly in-situ measured LAI is used for validating the 4-day time-series of Landsat-scale LAI. The importance of STARFM parameter settings and the spatial resolution (250 versus $1000 \mathrm{~m}$ ) of the MODIS LAI data for achieving reliable high spatial resolution LAI continuously over the growing seasons are also assessed.

\section{Study site and data}

\subsection{Study site and in-situ LAI measurements}

An 11-year record (2002 - 2012) of in-situ LAI was acquired from three sites located at the University 121 of Nebraska-Lincoln Agricultural Research and Development Center near Mead, Nebraska (Fig. 1), via 122 the Ameriflux site and data exploration system (http://ameriflux.ornl.gov/). Ne1 and Ne2 are managed 123 by center pivot irrigation systems, whereas Ne3 is rainfed. Maize is grown continuously at Ne1, while $124 \mathrm{Ne} 2$ and Ne3 rotate between maize and soybean. Further details of the sites are provided in Verma et al. 


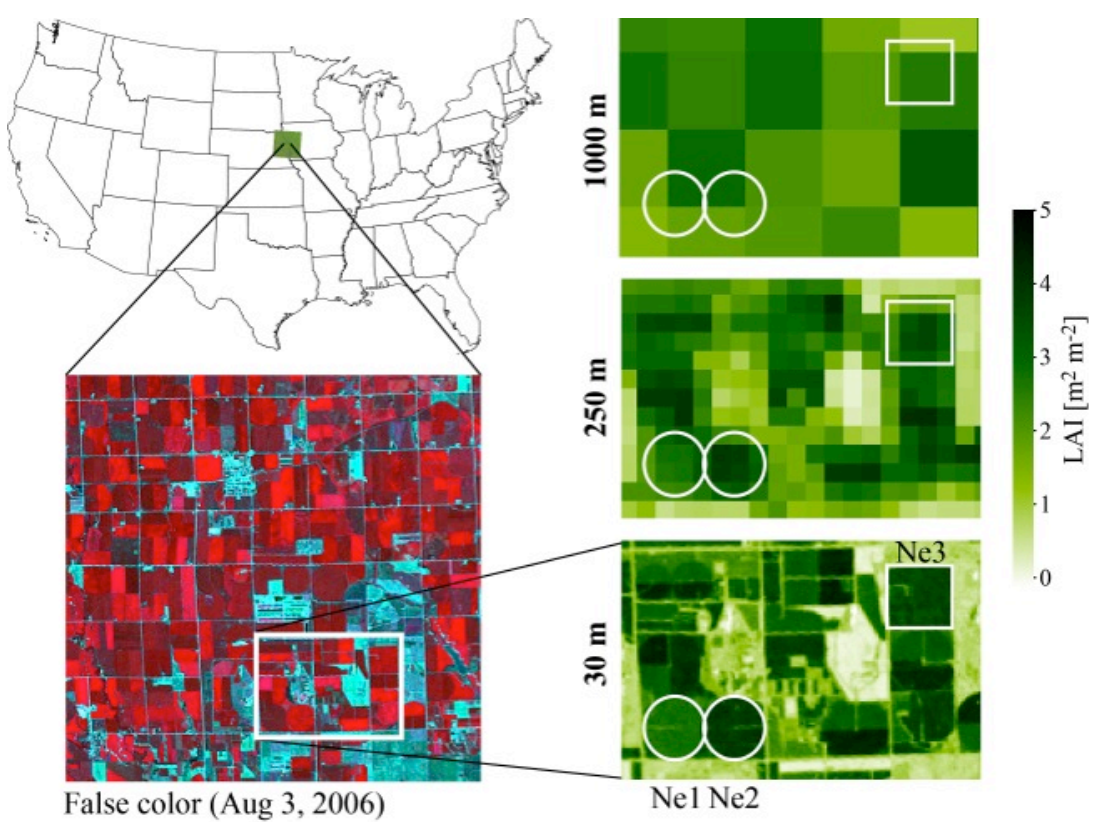

Fig. 1 Region of interest in Mead, Nebraska used for the testing of STEM-LAI. The subsets showcase multi-scale LAI retrievals over three field sites under continuous irrigated maize $\left(\mathrm{Ne} 1: 41.1645^{\circ} \mathrm{N}\right.$, $\left.96.4766^{\circ} \mathrm{W}\right)$ and irrigated $\left(\mathrm{Ne} 2: 41.1649^{\circ} \mathrm{N}, 96.4701^{\circ} \mathrm{W}\right)$ and rainfed $\left(\mathrm{Ne} 3: 41.1797^{\circ} \mathrm{N}, 96.4396^{\circ} \mathrm{W}\right)$ maize/soybean rotation.

125 (2005). Within each of the three fields, six small (20 x $20 \mathrm{~m})$ intensive measurements zones (IMZ) have 126 been established to represent intra-field variability and to allow accurate upscaling of ground 127 measurements to the whole-field level (Verma et al., 2005). Green LAI was determined from destructive 128 samples in each IMZ at approximately 7 to 10 day intervals over the full growing cycle, with data at the 129 six IMZs averaged to obtain field-wide representative values (Gitelson et al., 2003). The characteristic 130 phenology of in-situ measured LAI over the 2002-2012 period is depicted in Fig. 2, with the degree of 131 inter-annual variation defined by the shaded areas.

\subsection{Satellite data}

Medium resolution LAI inputs to STEM-LAI were provided via the 8-day combined Terra and Aqua collection 5 product (MCD15A2), produced at $1 \mathrm{~km}$ resolution from 2002 onwards. Relevant tiles were acquired for all 8-day compositing periods between days of year 100 and 300 over the 11-year study, using STEM-LAI built-in routines for automated download and post-processing, including stitching of MODIS tiles if the region of interest covers more than one tile. The MODIS LAI algorithm employs a biome-dependent look-up table approach based on simulations with a stochastic radiative transfer model 


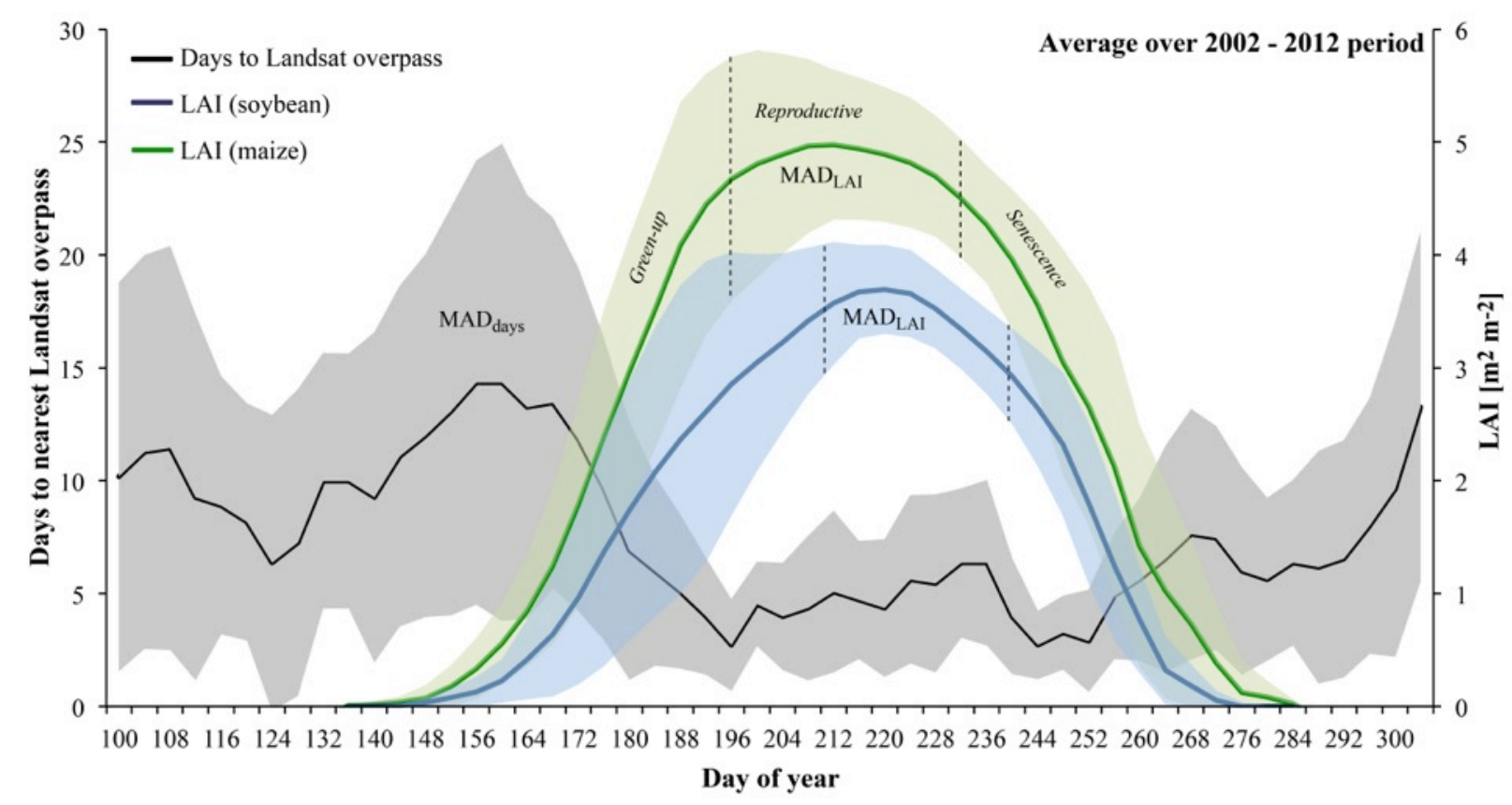

Fig. 2 Averaged time-series of the number of days to the nearest Landsat acquisition based on Landsat5 and Landsat-7 acquisition frequencies over the 11 year study period $(2002-2012)$. The grey-shaded area represents the Mean Absolute Deviation (MAD) and is an indication of inter-annual variation in the proximity to a Landsat acquisition on any given day. Year (2002-2012) averaged time-series of in-situ measured LAI over maize and soybean fields are depicted on the secondary y-axis.

141 that considers three-dimensional canopy structure (Huang et al., 2008). Prior information on soil patterns 142 and canopy structural attributes from 8 biome types (Knyazikhin et al., 1999) is used to constrain the 143 inversion process. If the primary algorithm fails, LAI is estimated using NDVI based on empirical 144 biome-specific relationships (Myneni et al., 1997; Knyazikhin et al., 1999). The 8-day Terra collection 5 145 surface reflectance product (MOD09Q1) that provides red and near-infrared band reflectances at $250 \mathrm{~m}$ 146 resolution, as well as yearly MODIS land cover type products (MCD12Q1), were also acquired as input 147 to STEM-LAI processing. Associated quality assurance information was obtained for assessing the 148 quality of the retrievals in the respective MODIS products.

150 All available Landsat-5 TM and Landsat-7 ETM+ scenes were downloaded from the Landsat archive 151 available on the Google cloud storage, amounting to a total of 142 Landsat scenes. All scenes have been 152 processed to Level 1T by the United States Geological Survey (USGS), ensuring systematic and 153 geometric accuracy by incorporating ground control points and a digital elevation model. Between 9 and 15416 Landsat scenes were acquired on an annual basis, with Fig. 2 displaying the number of days ( $\left.\mathrm{n}_{\text {days }}\right)$ to 155 the nearest Landsat overpass for each day, averaged over the 11-year study period. During most of the 
156 growing season, $\mathrm{n}_{\text {days }}$ averages to around 5 days for both maize and soybean. The largest intervals 157 between Landsat acquisitions occur in the spring (during early green-up) and late fall, due to more 158 frequent cloud cover and unstable atmospheric conditions during this period.

\section{Methods}

\subsection{Spatio-Temporal Enhancement Method for LAI (STEM-LAI)}

164 STEM-LAI embeds a two-step LAI downscaling technique (Section 3.1.1) and the STARFM data fusion 165 method (Section 3.1.2) within a completely automated processing framework (Fig. 3) for seamless 166 estimation of LAI at both high spatial $(30 \mathrm{~m})$ and temporal (4-day) resolution. All processing steps have 167 been streamlined for automatic execution on a Linux system and the approach has the capacity to be 168 implemented over any region of interest that has availability of both MODIS LAI and Landsat imagery. 169 STEM-LAI includes routines for on-the-fly download of relevant MODIS LAI and reflectance products from the Land Processes Distributed Active Archive Center (LP DAAC; http://e4ft101.cr.usgs.gov/) and available raw Landsat data $(5,7$ or 8$)$ are retrieved programmatically from the Google Cloud Storage. A scene cloud threshold of 50\% is enforced in the STEM-LAI Landsat acquisition scripts (based on cloud information in the associated metadata file) to limit low quality and cloud contaminated inputs. The Fmask object-based approach is adopted for cloud and cloud shadow detection in Landsat imagery (Zhu and Woodcock, 2012), and a second screening (40\% cloud threshold) is performed on co-registered time-series of cloud corrected (Fmask) Landsat image subsets for the given region of interest. Landsat digital counts are converted to directional surface reflectances using the Landsat Ecosystem Disturbance Adaptive Processing System (LEDAPS; Schmidt et al., 2013). STEM-LAI integrates Landsat-7 data despite striping effects due the scan line corrector failure (SLC-off) in May 2003 (Maxwell et al., 2007),

While the present implementation of STEM-LAI deals specifically with the integration of the MODIS LAI product, the approach can be extended to other operationally available medium resolution LAI products, providing a framework for multi-product downscaling of global medium resolution LAI for intercomparison and validation at high spatial resolutions $(30 \mathrm{~m})$. 


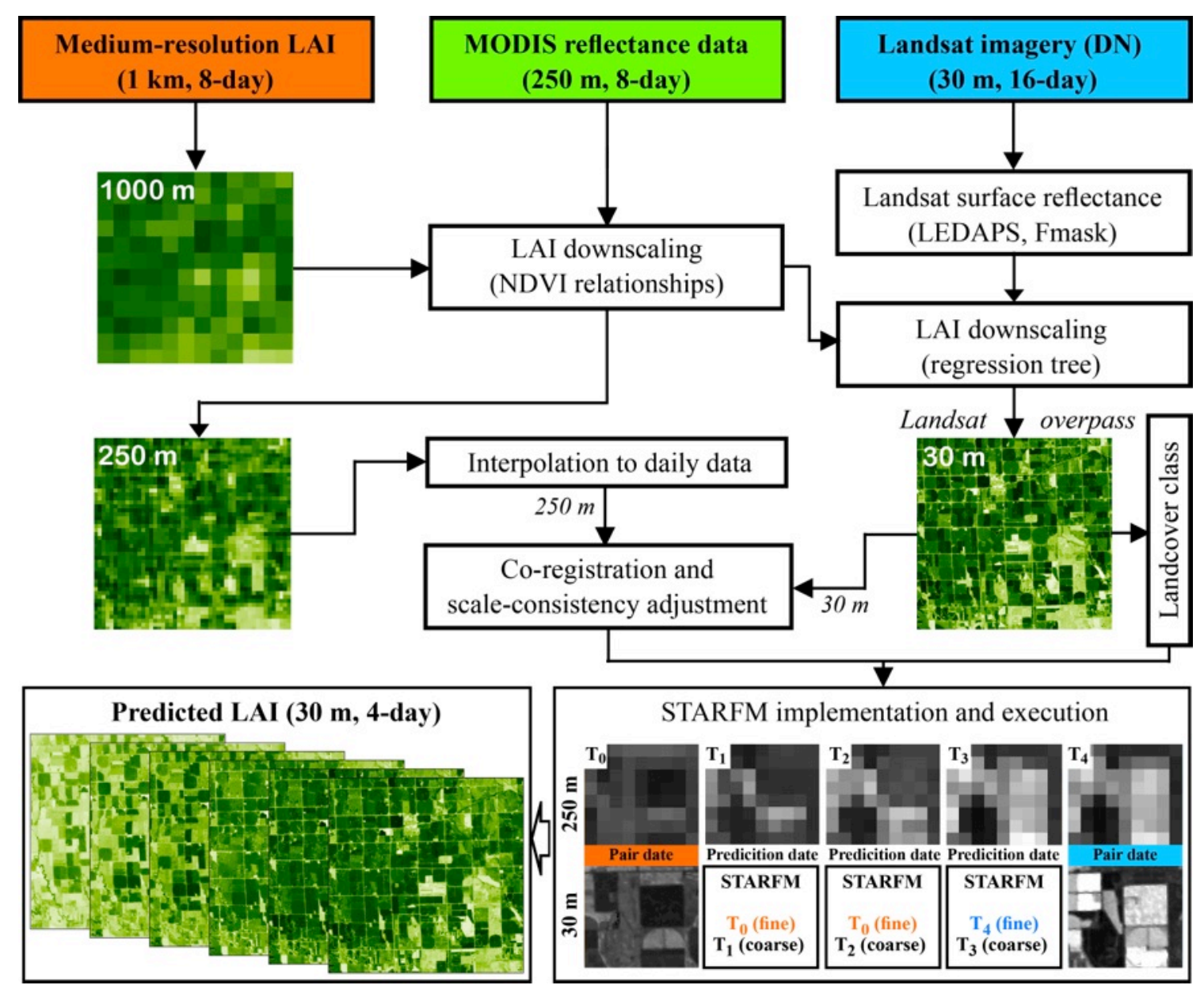

Fig. 3 Schematic diagram of the Spatio-Temporal Enhancement Method for LAI (STEM-LAI), depicting the processing steps required for predicting Landsat-scale LAI at 4-day intervals with input of medium-resolution LAI products and available Landsat imagery. The embedded STARFM combines medium and high resolution information from a pair date $\left(\mathrm{T}_{0}\right.$ or $\left.\mathrm{T}_{4}\right)$ with medium resolution (but high frequency) data from a prediction date $\left(\mathrm{T}_{1}, \mathrm{~T}_{2}\right.$ or $\left.\mathrm{T}_{3}\right)$ to derive high resolution data at that point (past or future) in time. All processes (including satellite data acquisition) have been streamlined for automatic execution on a Linux system.

\subsubsection{LAI downscaling}

189 The $1 \mathrm{~km}$ LAI data $\left(\mathrm{LAI}_{1 \mathrm{~km}}\right)$ is first downscaled to $250 \mathrm{~m}\left(\mathrm{LAI}_{250}\right)$ using a simple scale-consistent

190 NDVI-based approach in order to provide a better match to the typical dimension of agricultural field 191 boundaries (French, 2001). Doing this ensures that a sufficient number of homogeneous samples are 192 available for the regression tree training (described below) and STARFM fusion processes and is 193 expected to improve the STEM-LAI predictability over heterogeneous agricultural landscapes. The 194 downscaling of the 8-day composite $\mathrm{LAI}_{1 \mathrm{~km}}$ data to the $250 \mathrm{~m}$ scale uses as input the 8-day MODIS 250 195 m surface reflectance product (MOD09Q1) and the biome classification map (MCD12Q1) that forms the 
196 basis of the MODIS LAI/fPAR algorithm. For each 8-day MODIS composite, biome-specific LAI197 NDVI relationships are established at the $1 \mathrm{~km}$ scale by regressing high-quality MODIS LAI retrievals 198 against pixel-aggregated NDVI values calculated from high quality $250 \mathrm{~m}$ resolution surface 199 reflectances in the red $\left(\mathrm{R}_{\mathrm{red}}\right)$ and near-infrared $\left(\mathrm{R}_{\text {nir }}\right)$ wavebands (i.e. NDVI $=\left[\mathrm{R}_{\text {nir }}-\mathrm{R}_{\mathrm{red}}\right] /\left[\left[\mathrm{R}_{\text {nir }}+\mathrm{R}_{\mathrm{red}}\right]\right)$. 200 The regression process is informed and constrained by pre-established LAI-NDVI relationships derived 201 from radiative transfer model simulations (Knyazikhin, 1999; Myneni et al., 1997) to achieve a realistic 202 fit beyond the observed LAI range in the given MODIS composite. The resulting biome-specific LAI203 NDVI relationships are then applied at the $250 \mathrm{~m}$ resolution scale to calculate $\mathrm{LAI}_{250}$. Importantly, to 204 ensure exact scale consistency with the $\mathrm{LAI}_{1 \mathrm{~km}}$ retrievals, $\mathrm{LAI}_{250}$ is aggregated to the $1 \mathrm{~km}$ scale $205\left(\mathrm{LAI}_{1 \mathrm{~km} *}\right)$ and pixel-wise relative mean bias deviations between $\mathrm{LAI}_{1 \mathrm{~km}}$ and $\mathrm{LAI}_{1 \mathrm{~km} *}$ are applied 206 uniformly to $\mathrm{LAI}_{250}$ for all 16 sub-pixels. In this way, the LAI - NDVI relationships function as 207 determinants of sub-pixel (i.e. $250 \mathrm{~m}$ ) variability in LAI within a given $1 \mathrm{~km}$ pixel while ensuring pixel208 specific consistency with the original $\mathrm{LAI}_{1 \mathrm{~km}}$ when upscaled to the $1 \mathrm{~km}$ scale.

210 A regression tree approach (Gao et al., 2012) is then adopted for retrieving LAI from Landsat data. The 211 method uses high quality MODIS LAI retrievals from homogeneous (i.e. low sub-pixel variability) 212 pixels as reference samples to build rule-based regression models (using cubist from RuleQuest; 213 www.rulequest.com) that relate medium resolution LAI samples to aggregated (i.e. average of all pixel 214 values that contribute to the medium resolution output pixel) Landsat surface reflectances via multi215 variate linear regression. The explanatory variables used to build the regression tree consist of Landsat 216 bands 2-5 (i.e. green, red, near-infrared and mid-infrared) and NDVI, with multi-temporal MODIS and 217 Landsat image pairs for a given year incorporated into the process to include a wider range of sampled 218 LAI values. Training samples are taken from full Landsat scenes in order to obtain a sufficient number 219 for constructing the tree. The maximum number of rules (i.e. regression models) is fixed at 10 to ensure 220 good prediction accuracies without disrupting the spatial LAI value continuity. A new regression tree 221 will be produced for each growing season or year and the derived regression trees can then be applied to 222 all Landsat scenes to produce LAI values at $30 \mathrm{~m}$ resolution over the region of interest. Other settings 223 associated with the rule-based regression tree were defined in accordance with Gao et al. (2012). This 224 approach has been shown to produce high resolution maps of LAI that are generally consistent with the 225 medium resolution LAI product (Gao et al., 2012). 
227 Only MODIS LAI retrievals flagged as best quality (i.e. main radiative transfer algorithm and no 228 spectral signal saturation) are used in the regression tree training. Another fundamental requirement is 229 the existence of relatively homogenous pixels at the coarser scale, which implies low sub-pixel 230 variability in surface reflectances as determined from $30 \mathrm{~m}$ resolution Landsat data. The conditional 231 requirements (homogeneous samples with best quality flag) can limit the availability of samples for 232 certain cover types and environments, depending on land surface heterogeneity and characteristic field 233 size. In addition, the range of LAI appearing at the Landsat scale may differ from the range represented 234 by the selected MODIS samples, causing under-sampling of particularly high LAI values (Gao et al., 235 2012). Gao, Anderson, Kustas, \& Houborg (2014) demonstrated that the addition of information from 236 in-situ LAI measurements helped to train the regression models in cases when $1 \mathrm{~km}$ MODIS samples 237 were unable to represent high vegetation density features in Landsat data. While effective, this approach 238 relies on the availability of field measurements to support the training process, which is impractical for 239 applications aimed towards a more global implementation based on automated image-based 240 methodologies. A key refinement in this study is the use of $\mathrm{LAI}_{250}$ rather than $\mathrm{LAI}_{1 \mathrm{~km}}$ as input to the 241 regression tree, which is expected to provide a better record of LAI over the full range occurring at the 242 Landsat scale and to increase the number of homogeneous LAI samples.

\subsubsection{STARFM implementation and execution}

STARFM (Gao et al., 2006) capitalizes on the unique spatio-temporal characteristics of different satellite data streams and has previously demonstrated an ability to blend aspects of high spatial resolution Landsat and high temporal resolution MODIS surface reflectance data (Gao et al., 2006; Hilker et al., 2009; Wang et al., 2014). Recent efforts have extended STARFM to biophysical properties such as NDVI, evaporation and land surface temperature (Tian et al., 2013; Cammalleri et al., 2014; Weng et al., 2014). Here we apply the STARFM approach to LAI data for the first time by fusing co-

254 The data fusion process integral to STARFM is based on a weighting function that exploits information 255 from neighboring pixels and takes into account spatial, temporal and magnitude similarities between 256 high and medium resolution data streams (Cammalleri et al., 2014). In principle, high resolution data 257 can be predicted at any point in either the future or the past, with the availability of a medium resolution 
258 image at the prediction date and a pair of medium and high resolution images from a common time-step 259 (i.e. the pair date), although the process will benefit from multiple image pairs distributed over the 260 growing cycle (Gao et al., 2006). As indicated in previous studies (Cammalleri et al., 2014; Hilker et al., 261 2009) prediction accuracies may degrade when the range of conditions evident at the fine scale is not 262 well represented at the coarser scale. In this study, the use of $250 \mathrm{~m}$ resolution LAI data in the blending 263 process is expected to improve the ability of STARFM to reproduce high spatial resolution LAI 264 temporal dynamics, particularly in heterogeneous fine-grained landscapes or in regions with large 265 contrasts in LAI.

STEM-LAI implements a number of processing steps to ensure that the input data streams to STARFM are directly comparable and spatially and temporally consistent. The Landsat LAI scenes are first resized and co-registered to a given region of interest based on automated pre-processing routines embedded in the regularized canopy reflectance model, REGFLEC (Houborg et al., 2015b). The medium resolution 8-day LAI products are linearly interpolated to a daily time-scale, assuming LAI retrievals represent the mid-point of the compositing period. This may not always be the case, as it will depend on vegetation development and the daily data quality over the 8-day compositing period. Data are then re-projected from the MODIS sinusoidal projection to the Landsat UTM projection and resampled (nearest neighbor) to the $30 \mathrm{~m}$ Landsat resolution. This process produces day-specific data needed for the Landsat-MODIS data fusion and also fills any existing data gaps in the MODIS products. However the final high resolution LAI product is currently produced at 4-day intervals to reduce the computational demand of running STARFM and better reflect the native frequency of the input MODIS data streams. In order to minimize geolocation mismatches between Landsat and MODIS imagery, optimal co-registration is achieved by applying Landsat pixel shift distances (in $\mathrm{x}$ and $\mathrm{y}$ direction) that are identified based on the maximum correlation coefficient between Landsat and MODIS LAI for different $30 \mathrm{~m}$ pixel shifts (Wang et al., 2014). The pixel shifting step is critical to avoid confusing the

LAI algorithm and retrieval differences may also affect input data consistency. Landsat LAI ( $\left.\operatorname{LAI}_{30}\right)$ MODIS retrievals when aggregated to the $1 \mathrm{~km}$ scale (Gao et al., 2012). However, significantly larger discrepancies have been observed for saturated or low-quality MODIS LAI retrievals (Gao et al., 2012), 
as these are not included in the regression tree training process. In order to ensure consistency across spatial scales, 1) $\mathrm{LAI}_{30}$ is aggregated to the medium resolution $(250 \mathrm{~m})$ during Landsat acquisitions, 2) pixel-specific biases between Landsat pixel-aggregated and MODIS LAI $_{250}$ are recorded for each Landsat scene, and 3) biases are linearly interpolated to the daily scale and applied to daily $\mathrm{LAI}_{250}$ data. Apart from ensuring consistency during Landsat acquisitions, this process also provides a simple mechanism for extrapolating observed biases between Landsat acquisitions.

In STEM-LAI, STARFM predicts LAI at the Landsat spatial resolution every fourth day using input from near-coincident MODIS and Landsat LAI acquisitions (i.e. the pair date) and a MODIS LAI image from the prediction date (Fig. 3). Every fourth day, three plausible Landsat-MODIS image pairs are identified, based on their temporal proximity to the day of interest. The optimal pair is determined based on the maximum correlation between all pixels in MODIS images on the pair dates and the prediction date (Hilker et al., 2009; Cammalleri et al., 2014). Usually, this image pair predicts LAI for most Landsat pixels. However, cloud cover or SLC-off stripes (Landsat-7) in the Landsat LAI from the pair date will result in Landsat pixels without a prediction value. In this case, the pair with the next best correlation is used, and if gaps still remain, the last pair is used. In most situations, the automated stepwise image pair selection strategy will ensure high resolution LAI prediction without gaps with a frequency of 4 days. In order to avoid step changes in LAI (particularly across SLC-off stripes) resulting from the use of different MODIS-Landsat pairs, land cover specific adjustment factors calculated from pixel-averaged bias deviations between date-coincident predictions from the optimal and alternative pair dates, are applied to those pixels filled using the second and third pair date. Using time-series Landsat LAI images as input, the land cover classification is generated automatically based on a scheme that performs initial class separation (50 - 100 classes) via the unsupervised ISODATA technique, followed by class merging based on phenological similarity (Houborg et al., 2015b). The scheme ensures that ISODATA classes with similar phenology, as determined by the coefficient of efficiency (Section 3.3) computed between class-averaged LAI time-series, are grouped together.

\subsection{STARFM parameterization}

The accuracy of STEM-LAI predictions is influenced by a set of tunable STARFM parameters and input options. The maximum search distance parameter $(w)$ defines the size (half width in meters) of the 
320 moving window that is considered when creating the weight function and searching for neighboring 321 homogeneous medium resolution pixels that exhibit LAI magnitudes similar to the high spatial 322 resolution target pixel. The search for similar neighbor pixels may be informed by an ancillary 323 unsupervised land cover classification (Section 3.1.2) or by an internal threshold method that identifies 324 pixels within the local moving window that are similar to the central pixel (Gao et al., 2006). For the 325 latter, the calculated thresholds are affected by the parameterization of the number of spectral slices $(s)$, 326 which provides an indication of the number of land cover classes in the image. The use of a higher $s$ 327 value implies a stricter criterion for selecting pixels within the local moving window that are similar in 328 magnitude to the central pixel (Gao et al., 2006).

The sensitivity of STEM-LAI to these settings is evaluated in Section 4.2 using inputs of both $\mathrm{LAI}_{250}$ and $\mathrm{LAI}_{1 \mathrm{~km}}$ to the blending process. For this purpose, $w$ is varied iteratively between $250-1500 \mathrm{~m}$ $\left(\mathrm{LAI}_{250}\right)$ or $1000-3000 \mathrm{~m}\left(\mathrm{LAI}_{1 \mathrm{~km}}\right)$. The internal threshold method is also sensitive to the setting of $s$ that is varied iteratively from $5-40$. Optimal parameter settings are then derived by maximizing the agreement between STEM-LAI predictions and actual Landsat LAI retrievals based on cases with varying durations between pair and prediction day.

\subsection{Performance evaluation}

The fidelity of STEM-LAI for reproducing spatial features and magnitudes recorded in Landsat LAI imagery is evaluated through pixel-wise comparison of predicted and actual Landsat LAI images. The evaluation considers different plant phenological stages and durations between the pair and prediction day (8-64 days). This involves running STEM-LAI with a date-coincident Landsat and MODIS LAI image pair together with a MODIS LAI image from the prediction date to simulate a derived Landsatscale LAI for that date. Prediction performances can then be assessed by comparing the synthetic (i.e. STARFM simulated) retrievals against actual Landsat LAI retrievals from the prediction date. The predictive power of STEM-LAI is evaluated using inputs of both $\mathrm{LAI}_{250}$ and $\mathrm{LAI}_{1 \mathrm{~km}}$ to the regression tree and STARFM blending process.

For comparison against in-situ collected data, near-coincident STEM-LAI retrievals were extracted from averaged $3 \times 3$ pixel $(90 \times 90 \mathrm{~m})$ boxes centered on each IMZ and averaged in space to obtain estimates 
351 consistent with the in-situ measurements. The prediction performance is assessed using standard 352 statistical metrics including the coefficient of determination $\left(\mathrm{r}^{2}\right)$, Root-Mean-Square-Deviation (RMSD), 353 Mean Bias Error (MBE) and coefficient of efficiency $(E) . r^{2}$ represents a traditional correlation-based 354 metric that describes the proportion of the observed variance that can be explained by the model, 355 although a high goodness-of-fit is achievable even when large biases exist between the observed $(O)$ and 356 simulated $(P)$ data. $E$ has been described as an improvement over $r^{2}$ (Legates and McCabe, 1999) as it 357 takes into account the variability of the observed data according to

$E$ varies from 1 (perfect fit) to $-\infty$, where a value of zero indicates that the squared difference is as large as the variability in the observed data.

\section{Results and Discussion}

\subsection{Prediction of spatial patterns in actual Landsat LAI}

STEM-LAI facilitates downscaling of MODIS LAI ${ }_{1 \mathrm{~km}}$ to the $250 \mathrm{~m}\left(\mathrm{LAI}_{250}\right)$ and $30 \mathrm{~m}\left(\mathrm{LAI}_{30}\right)$ scale as exemplified in Fig. 4 for two Landsat acquisition dates separated by 16 days. Here, LAI $_{30}$ was generated using rule-based multi-variate regression models with input of MODIS LAI ${ }_{250}$ downscaled from $\mathrm{LAI}_{1 \mathrm{~km}}$ based on the scale consistent NDVI-based approach (Section 3.1.1). $\mathrm{LAI}_{30}$ resolves the fine-scale features of individual fields and crop pivots with a clear identification of field boundaries. General spatial LAI patterns remain visible in $\mathrm{LAI}_{250}$, but are largely indiscernible in $\mathrm{LAI}_{1 \mathrm{~km}}$. The frequency distributions (Fig. 4) indicate an increased clustering towards intermediate LAI with decreasing spatial resolution.

Fig. 5 visualizes the agreement between predictions and actual Landsat LAI on August $3^{\text {rd }} 2006$, based on Landsat-MODIS pair data from July $18^{\text {th }} 2006$ (Fig. 4a) and MODIS LAI on the prediction date (Fig. 4b). Results are shown using input of both $\mathrm{LAI}_{250}$ (Fig. 5b) and $\mathrm{LAI}_{1 \mathrm{~km}}$ (Fig. 5c). During the 16 days between coincident pair and prediction dates, maize is in the reproductive stage with relatively minor changes in LAI, whereas significant green-up occurs in fields planted with soybean (Fig. 2 and 4). 

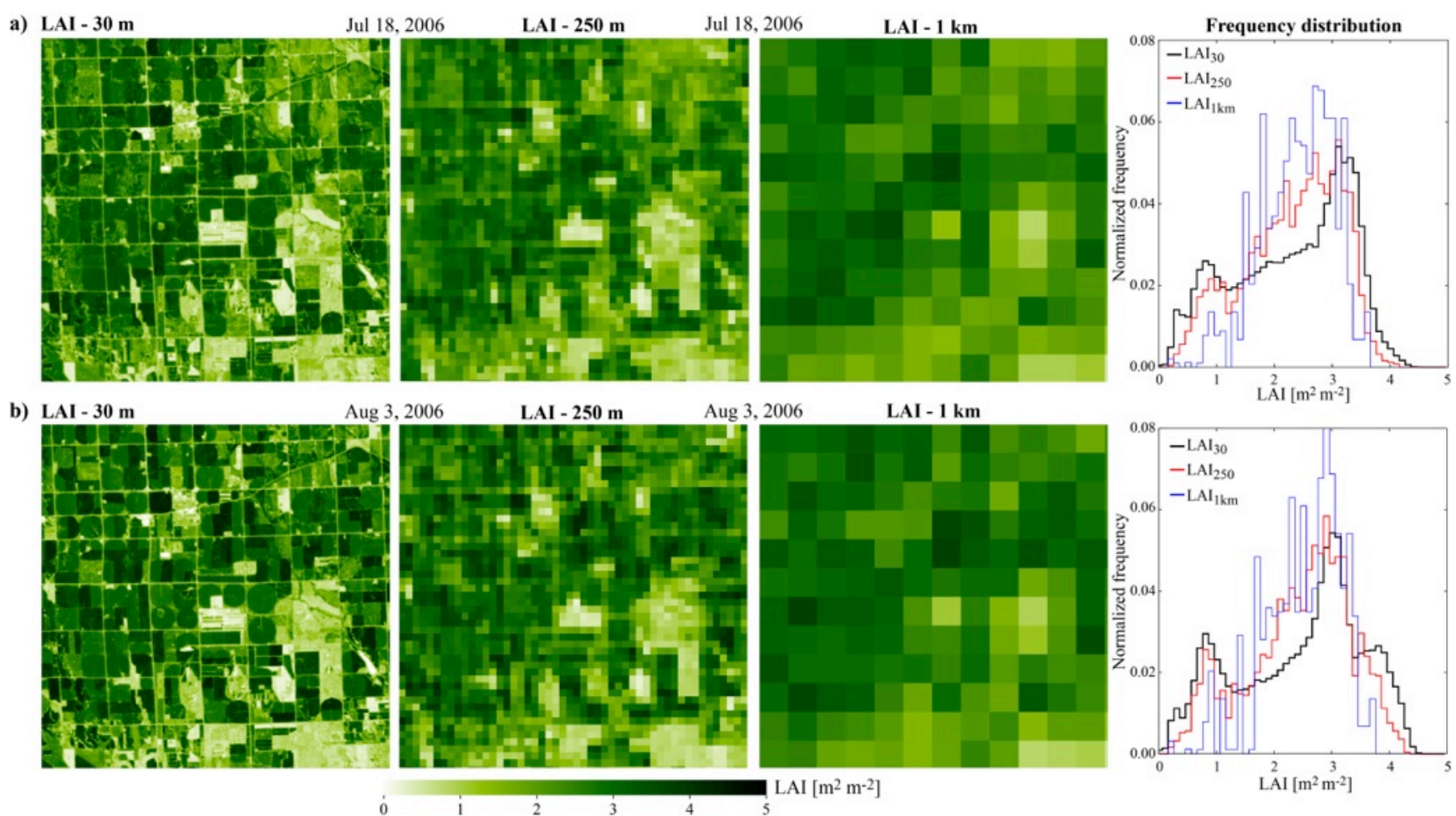

Fig. 4 Multi-scale (30, 250 and $1000 \mathrm{~m}$ ) maps of LAI during Landsat acquisitions on July 18 (a) and August 3 (b) 2006. The rightmost plot shows the normalized frequency distributions (i.e. probability density functions) of the three multi-scale LAI maps.

382 STEM-LAI 250 (Fig. 5b) is able to capture the majority of the observed LAI dynamics (Fig. 5a) as 383 corroborated by the statistical measures (Table 1) reporting an $\mathrm{r}^{2}$, RMSD, MBE, and $E$ of $0.9,0.34,0.01$, 384 and 0.9, respectively. Larger disagreements are evident between STEM-LAI $\mathrm{I}_{1 \mathrm{~km}}$ (Fig. 5c) and actual 385 Landsat LAI, with both $\mathrm{r}^{2}$ and $E$ decreasing significantly to 0.71 and the RMSD increasing to 0.65 386 (Table 1). The normalized frequency distributions (Fig. 5d) also show reduced capability of STEM$387 \quad \mathrm{LAI}_{1 \mathrm{~km}}$ in reproducing the lower and higher extremes in actual LAI.

389 The utility of STEM-LAI for reproducing spatial patterns and magnitudes recorded in Landsat LAI 390 imagery depends on the plant phenological stage and durations between the pair and prediction day (839164 days) (Table 1). As expected, prediction accuracies typically decrease with increasing number of 392 days between pair and prediction dates, but even with a time gap of 64 days, STEM-LAI 250 predictions 393 are able to explain $78 \%$ of the variance with an RMSD of 0.40 (Table 1). In contrast, STEM-LAI ${ }_{1 \mathrm{~km}}$ 394 largely fails to reproduce the Landsat scale LAI dynamics on this date, as indicated by very low 395 correlation statistics $\left(\mathrm{r}^{2}=0.09\right.$ and $\left.E=0.01\right)$. In all cases, the incorporation of $\mathrm{LAI}_{250}$ data in the STARFM 


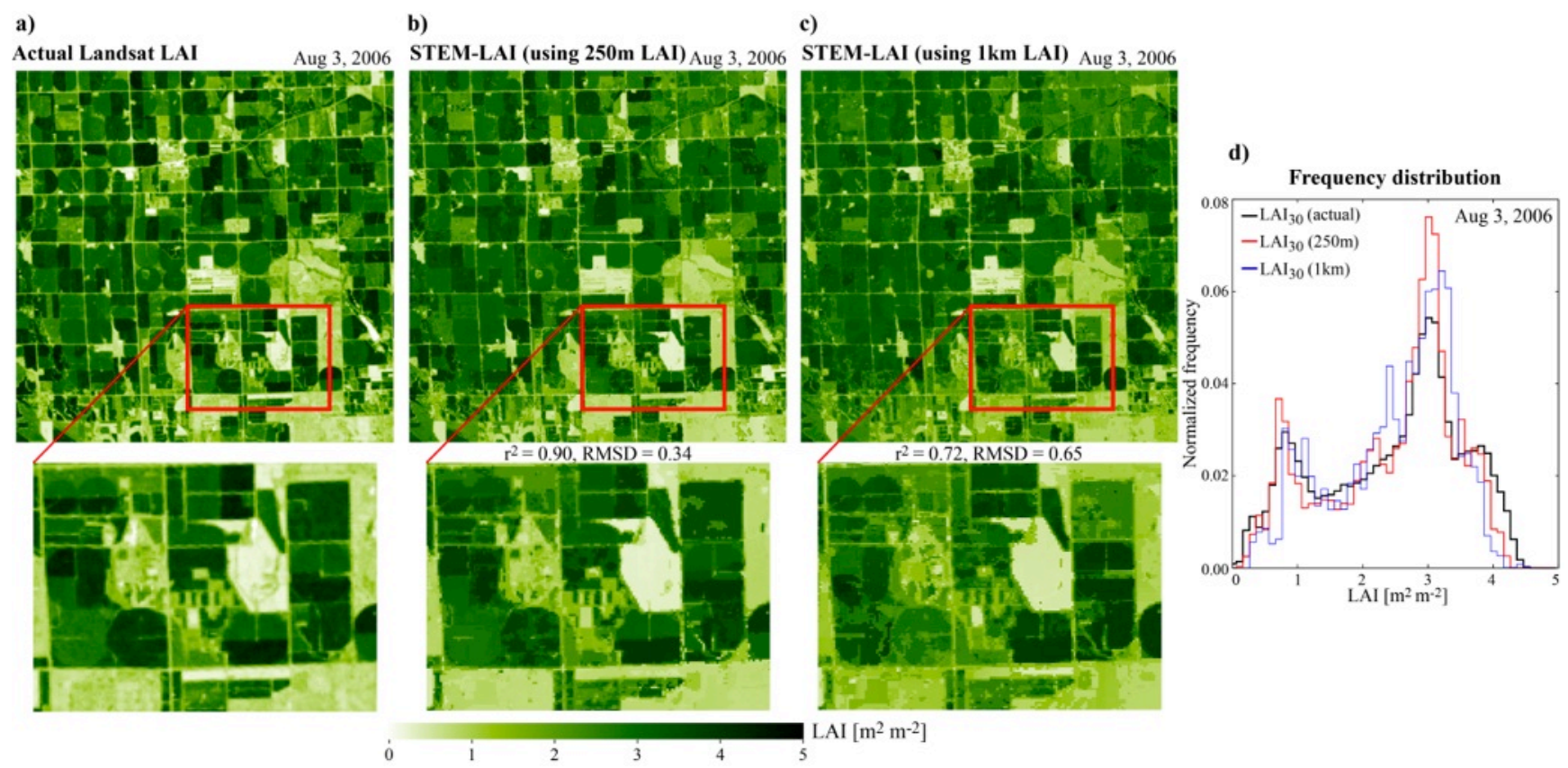

Fig. 5 Actual Landsat LAI on August 3, 2006 (a) and STEM-LAI predictions using input of both $\mathrm{LAI}_{250}$ (b) and $\mathrm{LAI}_{1 \mathrm{~km}}$ (c). The predicted images were based on MODIS - Landsat pair data from July 18, 2006 (Fig. 4a) and MODIS LAI on the prediction date (Fig. 4b). The lower panel displays zooms over the sites with in-situ LAI measurements. Normalized frequency distributions (pdfs) of actual and predicted $30 \mathrm{~m}$ LAI are intercompared in the rightmost plot.

396 fusion process demonstrates an improvement over the use of $\mathrm{LAI}_{1 \mathrm{~km}}$, with enhanced capacity to 397 reproduce fine-scale LAI features recorded in actual Landsat LAI imagery (Table 1). These observations 398 concur with Cammalleri et al. (2014), who reported issues in reproducing spikes in observed ET when 399 running STARFM with $1 \mathrm{~km}$ MODIS inputs, as the coarse MODIS pixel scale did not properly resolve 400 irrigation events. It should be noted that actual prediction accuracies over the majority of the growing 401 season will generally be better than indicated in these examples, as the daily proximity to the nearest 402 valid Landsat acquisition typically averages around 5 days for the 11 growing seasons (Fig. 2).

\subsection{STARFM parameter optimization}

As noted in Section 3.2, the STARFM predictions are affected by the number of spectral slices $(s)$, the maximum search distance $(w)$, and the method used to inform the search for similar neighbor pixels. The setting of $w$ can be important when the ancillary land cover classification method is adopted for searching for similar neighbor pixels. For example, changing $w$ from 250 to $1500 \mathrm{~m}$ in 2003 caused the relative RMSD (rRMSD) between actual LAI and STEM-LAI 250 predictions to decrease from $47 \%$ to 
Table 1 Statistic measures of the ability of STARFM to predict spatial patterns and magnitudes of LAI as recorded in actual Landsat LAI images. Coincident Landsat and MODIS LAI images at the pair date (PairD) and MODIS LAI acquired at prediction date (PredD) were used to predict LAI at Landsat spatial resolution at PredD for different durations between pair and prediction day ( $8-64$ days). Results are reported for MODIS $\mathrm{LAI}_{250}$ and $\mathrm{LAI}_{1 \mathrm{~km}}$ (in parentheses) inputs to STARFM, and were based on the land cover classification method for informing the search for similar neighbor pixels using optimal settings for the maximum search distance $(w)$.

$\mathrm{O}$ is the mean of the in-situ measurements, RMSD is the root-mean-square difference between model estimates and measurements, $\mathrm{MBE}$ is the mean bias error (if positive measurements are being overestimated), $\mathrm{r}^{2}$ is the coefficient of determination, and $E$ is the coefficient of efficiency.

\begin{tabular}{llllllllll}
\hline Year & PairD & PredD & days & $w[\mathrm{~km}]$ & $O$ & $r^{2}$ & $R M S D$ & $M B E$ & $E$ \\
\hline 2002 & Jul 15 & Jul 23 & 08 & $0.75(3)$ & 1.96 & $0.95(0.84)$ & $0.26(0.42)$ & $0.01(0.03)$ & $0.94(0.81)$ \\
2003 & Jul 10 & Sep 12 & 64 & $1.5(3)$ & 1.23 & $0.78(0.09)$ & $0.40(0.77)$ & $0.03(0.09)$ & $0.77(0.01)$ \\
2004 & Jul 12 & Aug 13 & 32 & $1.5(3)$ & 2.75 & $0.87(0.57)$ & $0.45(0.83)$ & $-0.02(-0.04)$ & $0.87(0.56)$ \\
2005 & May 28 & Jun 13 & 16 & $0.5(3)$ & 0.99 & $0.77(0.47)$ & $0.28(0.41)$ & $-0.01(-0.01)$ & $0.77(0.46)$ \\
2006 & Jul 18 & Aug 3 & 16 & $0.75(1.5)$ & 2.50 & $0.90(0.72)$ & $0.34(0.65)$ & $0.01(-0.07)$ & $0.90(0.71)$ \\
2007 & Jul 5 & Jul 21 & 16 & $0.75(2.5)$ & 2.40 & $0.88(0.74)$ & $0.34(0.53)$ & $0.01(0.01)$ & $0.88(0.74)$ \\
2008 & Aug 8 & Sep 9 & 32 & $1.5(3)$ & 1.71 & $0.83(0.67)$ & $0.35(0.60)$ & $0.02(0.03)$ & $0.83(0.67)$ \\
2009 & Jul 26 & Sep 4 & 40 & $1.5(3)$ & 2.61 & $0.77(0.50)$ & $0.39(0.87)$ & $-0.04(-0.09)$ & $0.76(0.48)$ \\
2010 & Aug 30 & Sep 7 & 08 & $0.25(1)$ & 1.53 & $0.90(0.86)$ & $0.29(0.49)$ & $0.00(0.00)$ & $0.90(0.85)$ \\
2011 & Jun 30 & Jul 16 & 16 & $1.5(2)$ & 2.72 & $0.81(0.60)$ & $0.52(0.74)$ & $-0.02(-0.01)$ & $0.80(0.55)$ \\
2012 & Jul 26 & Jul 10 & 16 & $1.5(3)$ & 2.52 & $0.86(0.68)$ & $0.40(0.59)$ & $-0.01(-0.02)$ & $0.86(0.68)$ \\
\hline
\end{tabular}

$41133 \%$ (Fig. 6a). In most cases, $w=1000 \mathrm{~m}$ represents a reasonable parameterization for minimizing the 412 rRMSD when $\mathrm{LAI}_{250}$ is used as input, (Fig. 6a, Table 1), while $w=3000 \mathrm{~m}$ is an appropriate setting when 413 using $\mathrm{LAI}_{1 \mathrm{~km}}$ as input (Fig. 6b, Table 1).

415 The internal threshold method, which represents an alternative for identifying pixels within the local 416 moving window that are similar to the central pixel, is influenced by the setting of $s$ and is characterized 417 by significantly different sensitivities towards changes in $w$ (Fig. 6c). In all test cases, setting $w=250 \mathrm{~m}$ 418 produces the lowest rRMSD, with a clear tendency towards increasing rRMSD with increasing $w$. The 419 variation in prediction performance due to $s$ (indicated by the error bars in Fig. 6c) can be substantial 420 and typically a low value of $s(5-10)$ performs best for the reported durations between pair and 421 prediction date.

423 Fig. 7 compares LAI predictions from STEM-LAI implemented with the land cover classification and 424 internal threshold method, respectively, against actual Landsat LAI for three different prediction dates 
425 that are 64 days (Fig. 7a), 32 days (Fig. 7b) and 8 days (Fig. 7c) from the date of the Landsat-MODIS 426 image pair. Comparative normalized frequency distributions of actual and predicted $30 \mathrm{~m}$ resolution LAI 427 are included for each case, and scene-specific optimized parameter settings ( $s$ and $w$ ) are provided on the 428 plots along with standard statistical metrics. When the land cover classification is used, field boundaries 429 are well delineated and much of the sub-pivot LAI dynamics are captured, even when the prediction date 430 is far away from the Landsat-MODIS pair date (Fig. 7a). The internal threshold method has more limited 431 utility when the pair and prediction dates are far apart, with predictions tending to be characterized by a 432 smoothed appearance with poor delineation of field boundaries and record of sub-field heterogeneity 433 (Fig. 7a and b). In these cases the highest prediction accuracies are obtained with a low value of $s$ (5434 10). Increasing $s$ enhances the fine-scale detail and reduces the smoothed appearance, but at the expense 435 of a decreasing $\mathrm{r}^{2}$ and an increasing RMSD (Fig. S1).

437 With closer proximity to the pair date (8 days), the internal threshold method performs slightly better $438\left(r^{2}=0.92\right.$ and $\left.R M S D=0.27\right)$ than the land cover classification approach $\left(r^{2}=0.9, \operatorname{RMS}=0.29\right)$, although 439 the frequency distributions of both methods are a near perfect match to the distribution of the actual LAI 440 (Fig. 7c). The internal threshold method establishes neighboring pixel similarity based on scene-specific 441 conditions at the pair date, and assumes that these pixel associations also apply during the prediction 442 date, which is a reasonable assumption when close to the pair date. The land cover classification on the 443 other hand contains multiple established classes sharing pixel associations that extend over the full 444 growing season, and thus represent a mechanism for providing better informed predictions further away 445 from the Landsat-MODIS pair date.

447 With near-coincident pair and prediction date, the actual (Jul 18, 2006) and predicted (Jul 19, 2006) LAI 448 should provide a near-perfect match (Fig. S2). However this is only achieved when the internal 449 threshold method is enabled and $s$ is set to 40, resulting in an $r^{2}$ of 0.997 and relative RMSD of $2.9 \%$ 450 (Fig. S2c). A lower $s$ setting (5) causes a smoother appearance, as witnessed in previous examples and 451 the rRMSD increases to $6.8 \%$ (Fig. S2f). The agreement with the actual LAI is further reduced when 452 using the land cover classification approach, with $\mathrm{r}^{2}$ and $\mathrm{rRMSD}$ ranging between 0.92-0.95 and 9.6-12 $453 \%$ respectively, depending on the specification of $w$ (highest accuracy achieved with $w=250$ in this case) 454 (Fig. S2b and e). Clearly, the internal threshold method is superior when it comes to reproducing high 455 resolution intra-field features in LAI (Fig. S2d). However as mentioned above, the neighboring pixel 


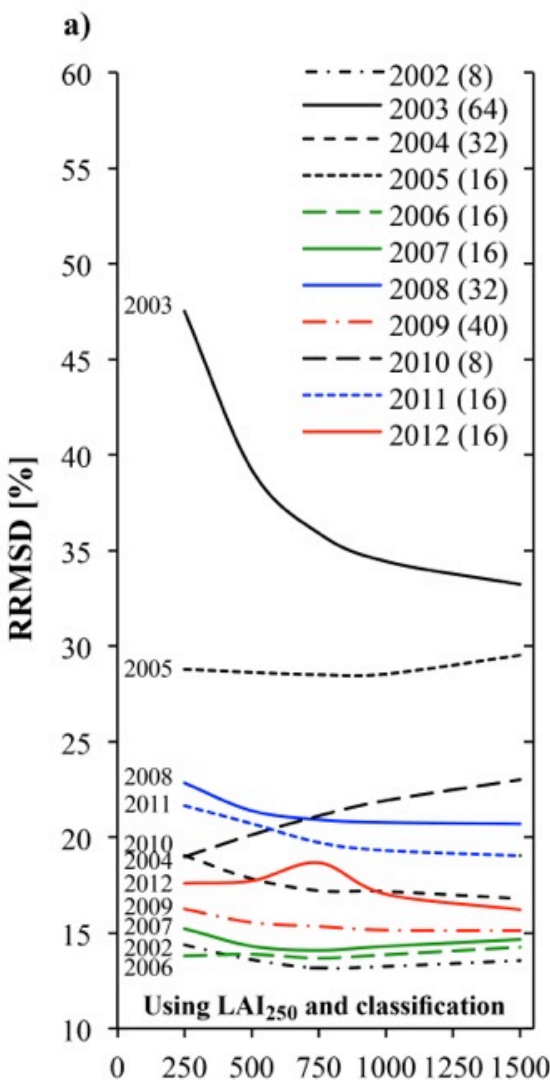

b)

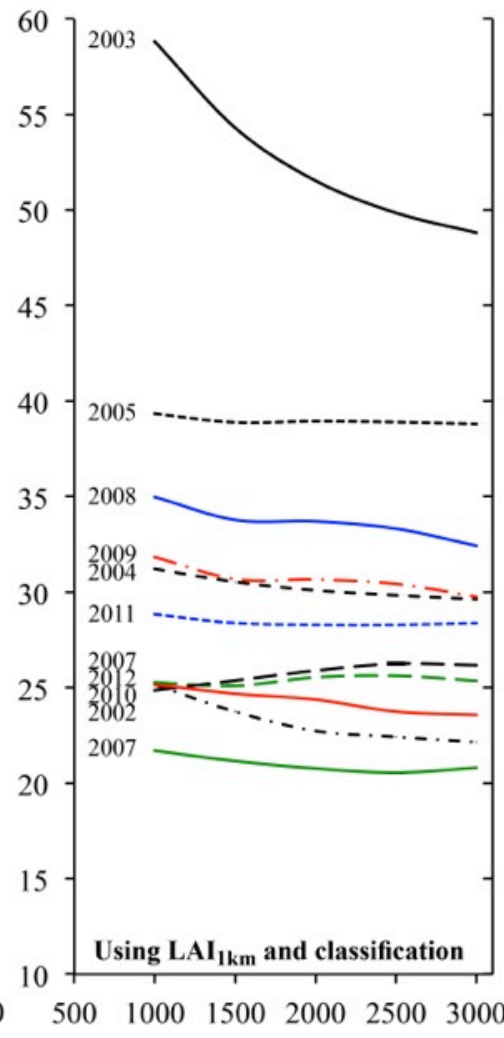

c)

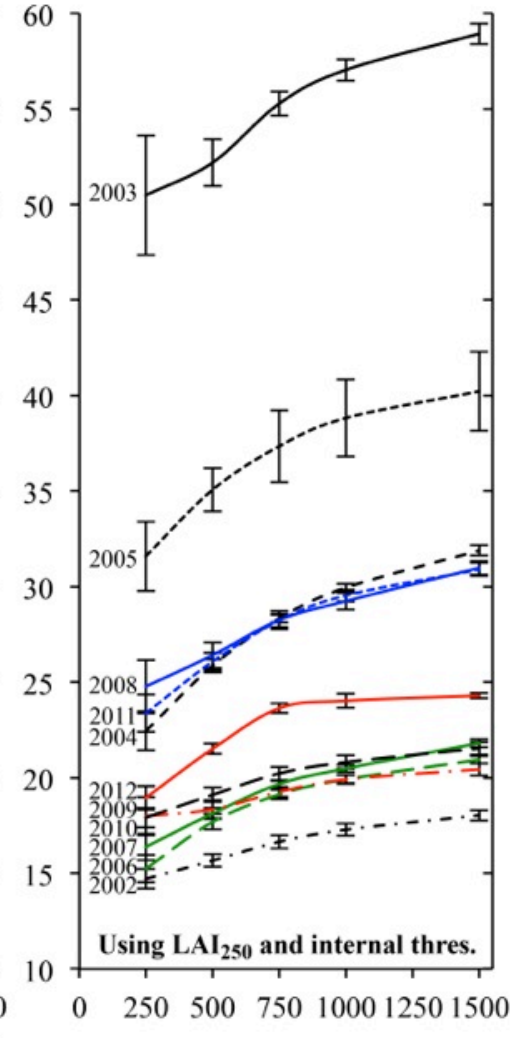

Fig. 6 The relative RMSD between actual Landsat LAI and STEM-LAI predictions as a function of the maximum search distance for the 11 test cases (the value in parentheses is the duration between pair and prediction date) shown in Table 1. Results are reported based on the land cover classification method with input of $\mathrm{LAI}_{250}$ (a) and $\mathrm{LAI}_{1 \mathrm{~km}}$ (b), and the internal threshold method with input of $\mathrm{LAI}_{250}$ (c). In c) the error bars represent variations in retrieval performance due to $s$ (number of spectral slices, 5-40).

associations established by this method have decreasing validity with increasing number of days between the pair and prediction date.

459 In summary, the threshold method works best for predictions close to the pair date (less than $\sim 8$ days), 460 while the land cover classification approach is favored for predictions further away from the pair date. 461 The settings for $w$ and $s$ depend on the resolution of the input medium resolution LAI (1 km or $250 \mathrm{~m})$ 462 and the adopted pixel similarity identification approach. Accordingly, the internal threshold method 463 should be applied when the distance to the pair date $\left(\mathrm{D}_{\text {pair }}\right)$ is less than 8 days, with $w$ set to either 250 or $4641000 \mathrm{~m}$ and $s$ decreasing gradually with $\mathrm{D}_{\text {pair }}\left(s=\left[8-\mathrm{D}_{\text {pair }}{ }^{* 5+5}\right)\right.$. The gradual decrease in $s$ with 465 increasing duration between the pair and prediction date will retain subtle intra-field features in LAI 

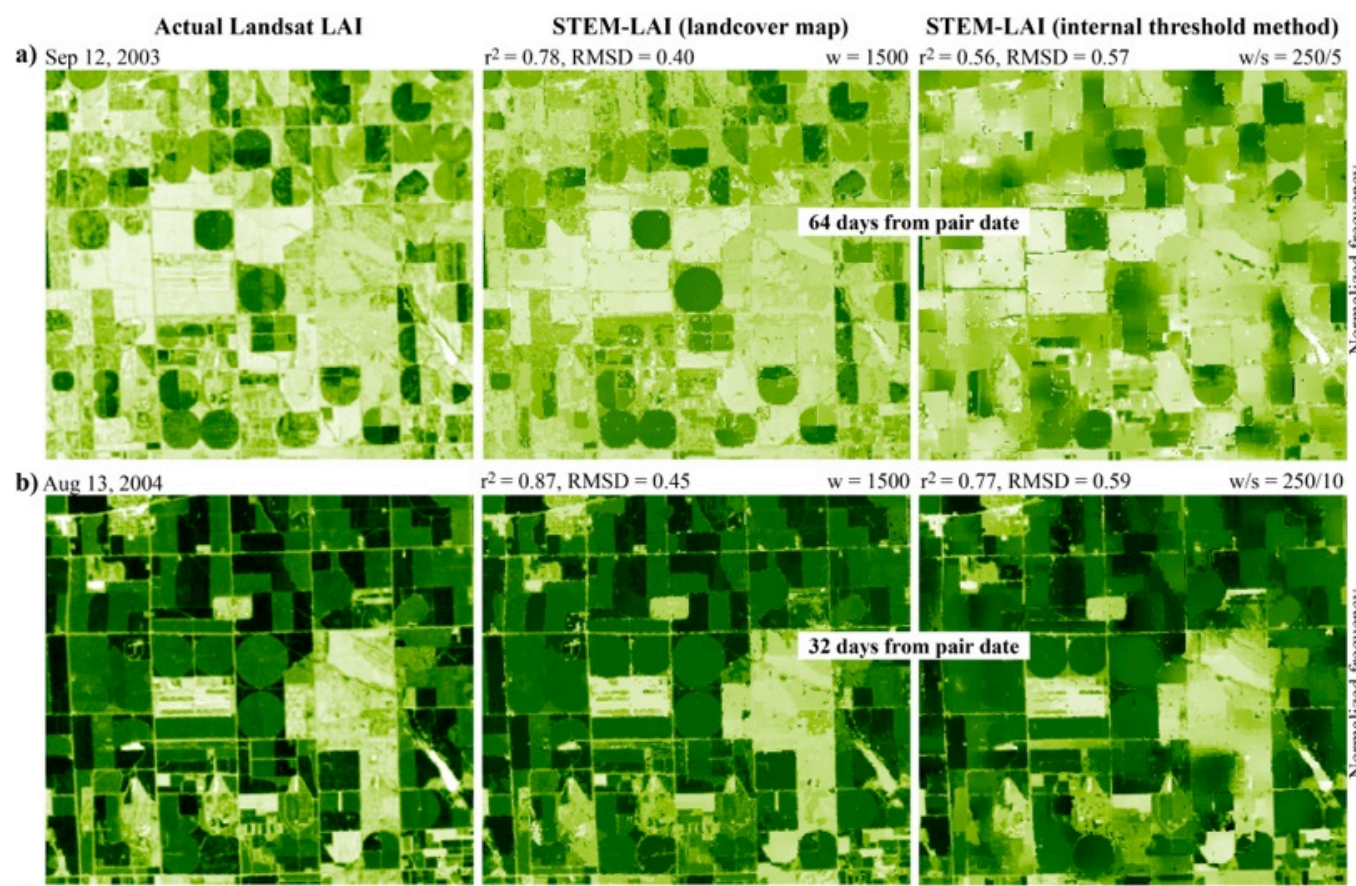

$\mathrm{w}=1500 \mathrm{r}^{2}=0.77, \mathrm{RMSD}=0.59$

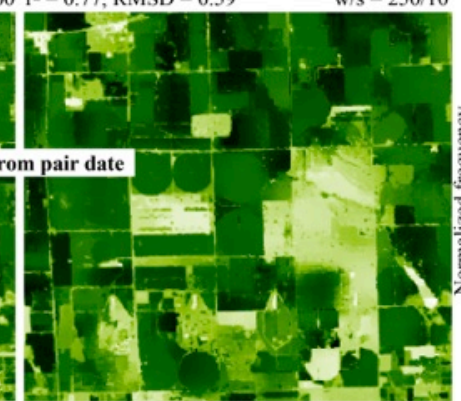

c) $\operatorname{Sep} 7,2010$
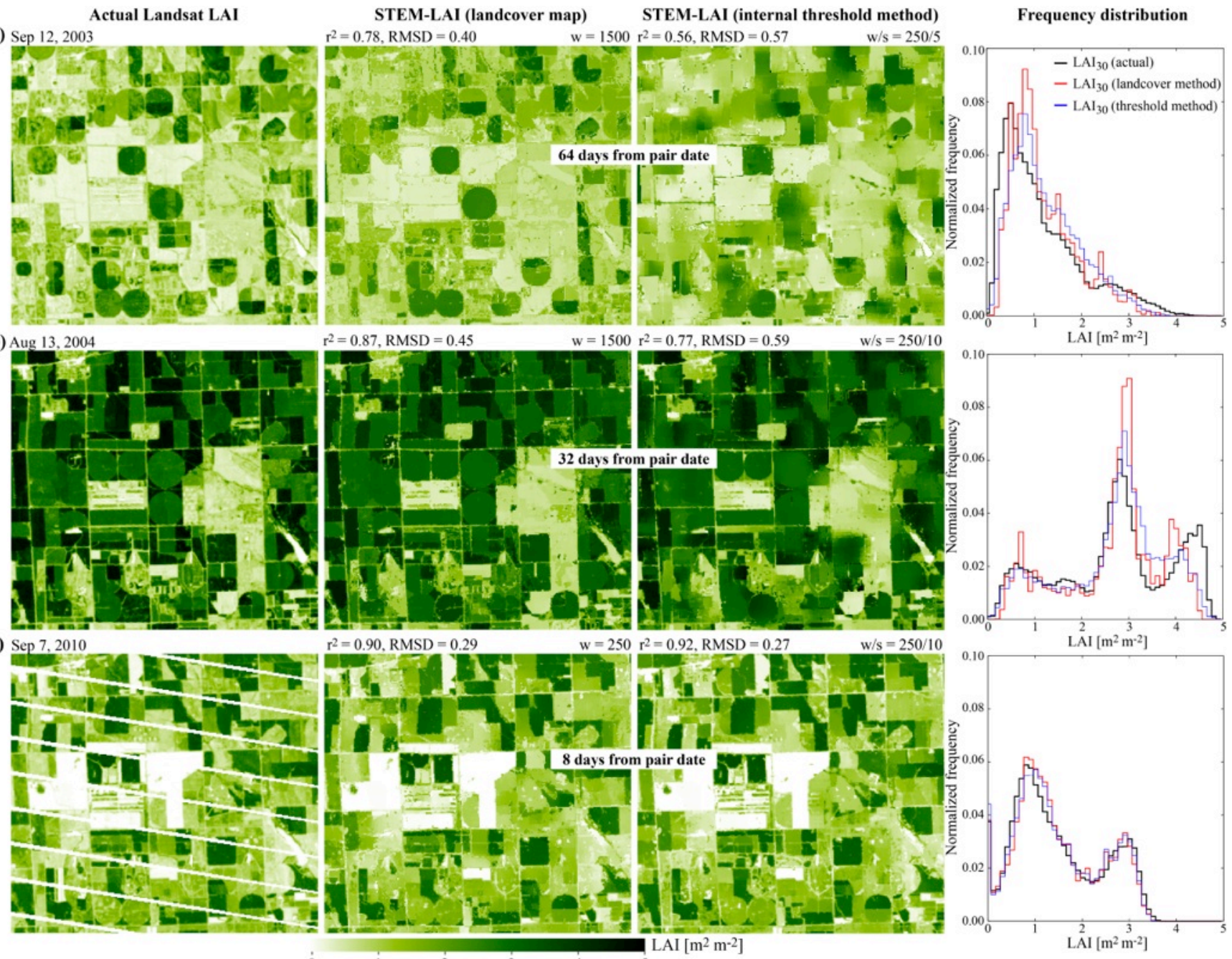

$=0.90, \mathrm{RMSD}=0.29$

$W=250 r^{2}=0.92$, RMSD $=0.27$
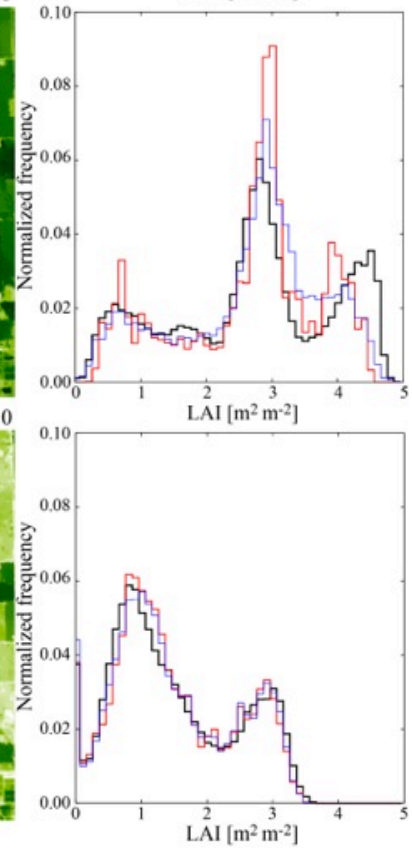

Fig. 7 Comparisons of actual Landsat LAI against STEM-LAI ${ }_{250}$ predictions using the land cover classification or internal threshold method with input of MODIS - Landsat image pairs 64 days (a), 32 days (b) and 8 days (c) from the prediction date. $\mathrm{R}^{2}$ and RMSD metrics along with optimal settings for $w$ and $s$ (i.e. those resulting in the highest values of $E$ ) are reported for each prediction image. Normalized frequency distributions (pdfs) of actual and predicted $30 \mathrm{~m}$ LAI are provided in the rightmost plots. Stripes occur in the actual LAI map in (c) due to the scan line corrector failure of the Landsat-7 ETM+ sensor in May 2003.

close to Landsat acquisitions, while maintaining good overall predictabilities further away from the Landsat-MODIS pair date. The ancillary land cover classification on the other hand is most appropriate when $\mathrm{D}_{\text {pair }}>7$, with $w$ set to either 1000 or $3000 \mathrm{~m}$ ( $s$ is not used in this case). These parameter settings are automated in STEM-LAI in order to enhance LAI predictabilities over a wide range of conditions and Landsat acquisition frequencies. 
a)
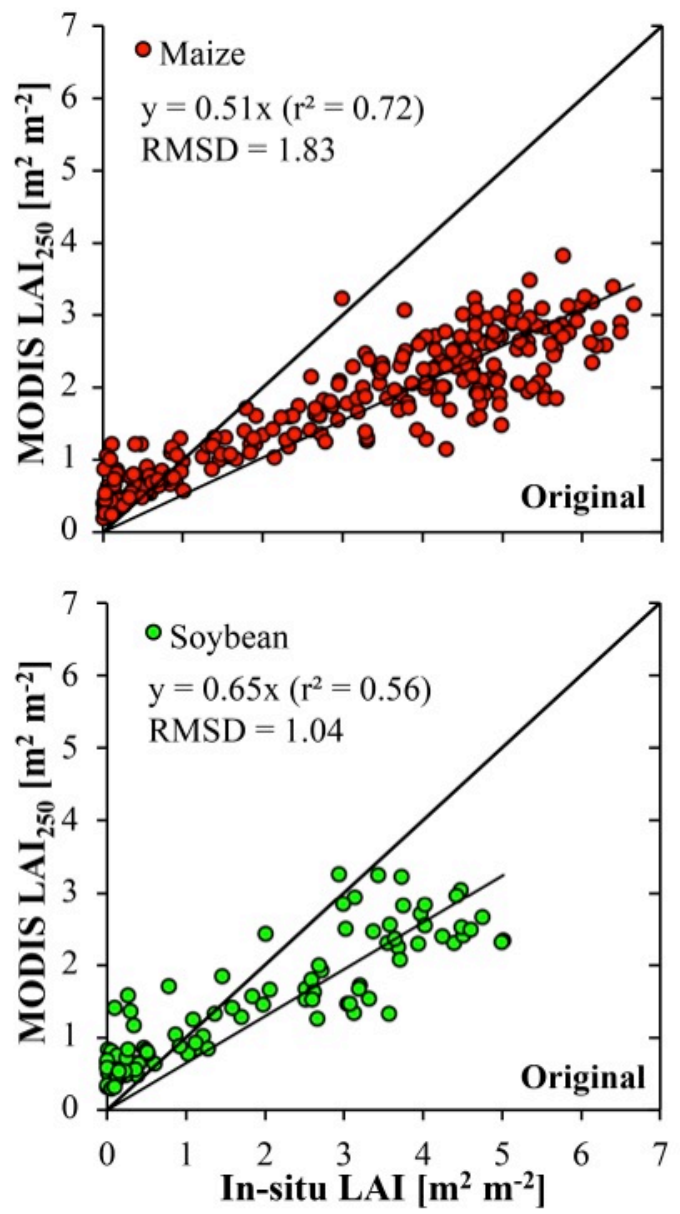

b)
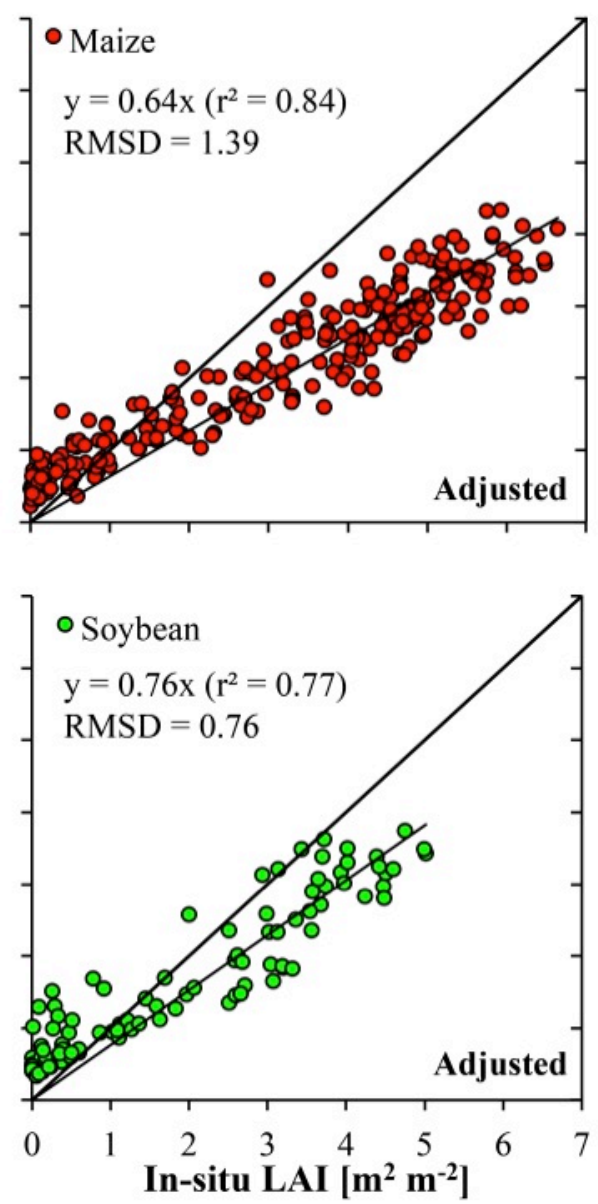

Fig. 8 LAI scatter plots comparing in-situ measured LAI in maize and soybean against date-coincident (i.e., linear interpolation of 8-day time-series) original MODIS $\mathrm{LAI}_{250}$ (a) and consistency adjusted $\mathrm{LAI}_{250}$ (b).

474 The agreement between the in-situ LAI record and downscaled $(250 \mathrm{~m})$ MODIS LAI is affected by the 475 bias and phenology adjustment approach (Section 3.1.2) that is adopted to ensure scale consistent data 476 streams as input to STARFM (Fig. 8). The use of the consistency adjusted dataset in place of the original 477 MODIS LAI 250 , results in $\mathrm{r}^{2}$ increasing from 0.72 to 0.84 for maize and from 0.56 to 0.77 for soybean 478 (Fig. 8). At the same time the RMSD values decrease substantially, a result in part of a bias reduction. 479 The improvement can be attributed to the fact that only high-quality MODIS retrievals are used in the 480 training process for establishing the rule-based regressions to be applied to the full Landsat scene, 481 thereby avoiding the added uncertainties associated with lower quality MODIS retrievals. 
a)
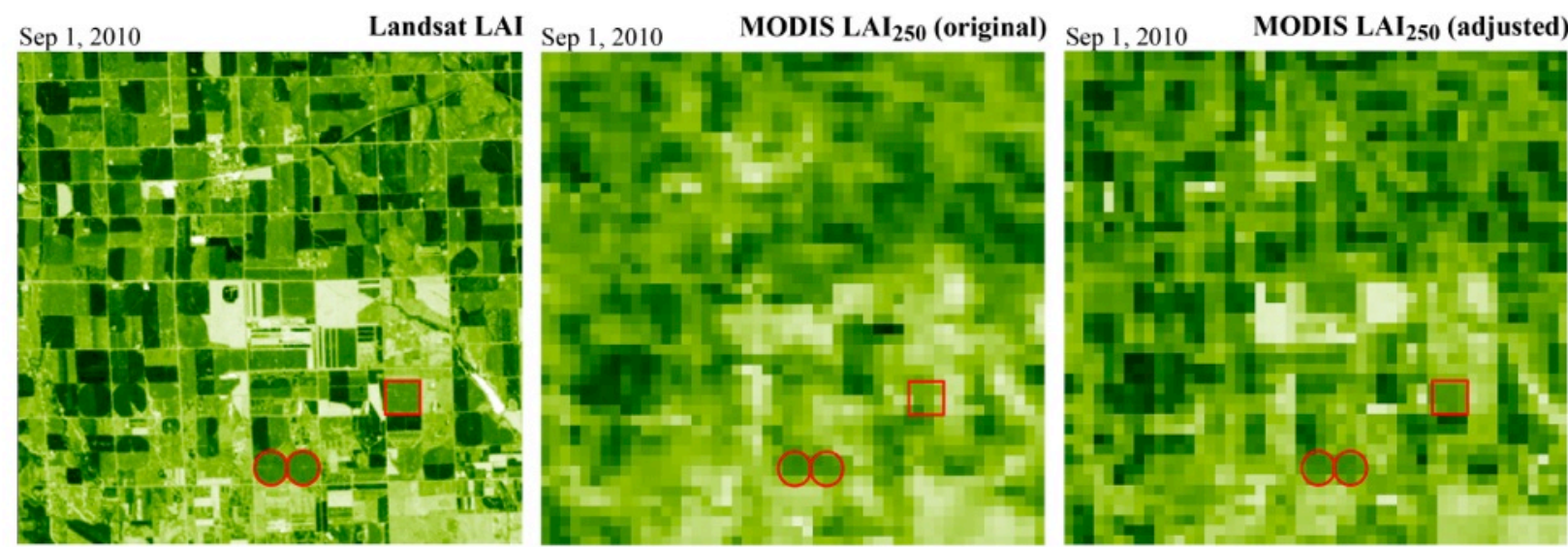

b)
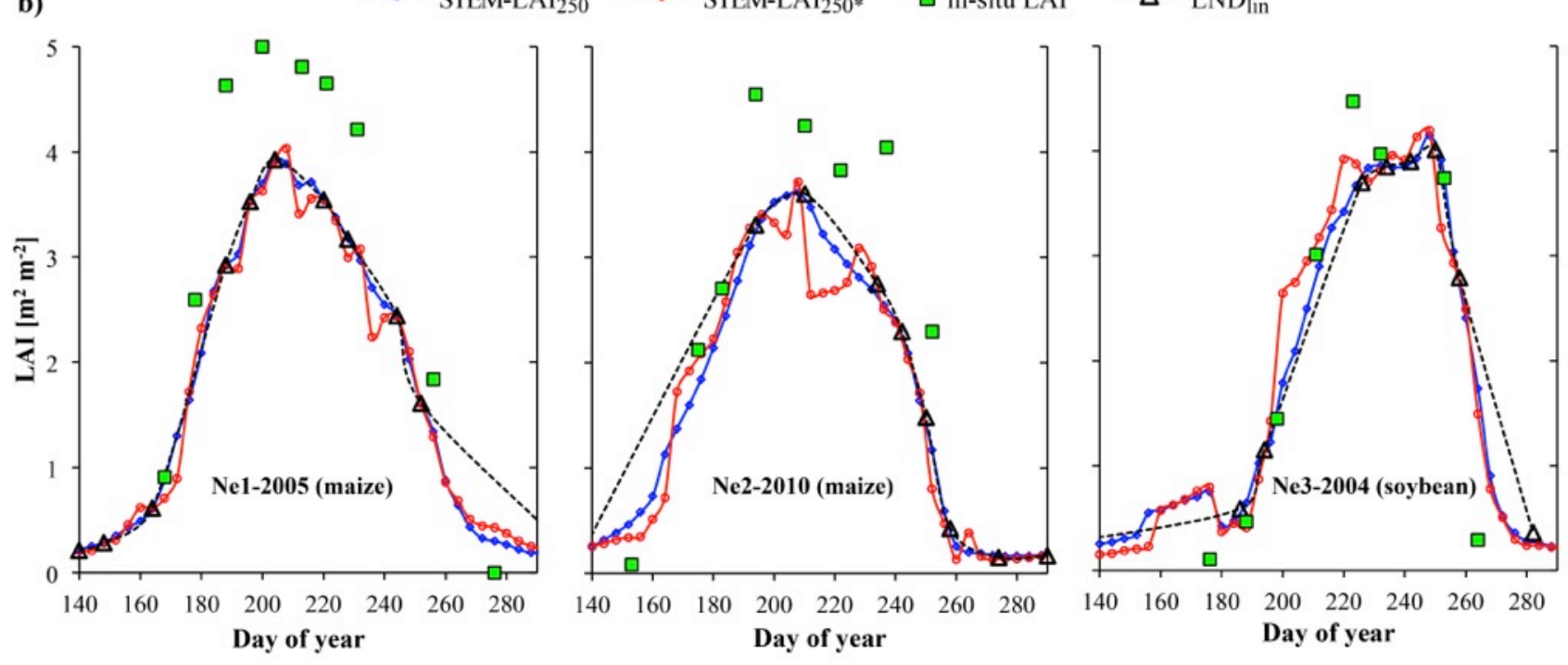

Fig. 9 (a) Visualization of differences between the original and adjusted MODIS LAI 250 images for a Landsat overpass date. (b) LAI time-series for field Ne1 (maize), Ne2 (maize) and Ne3 (soybean) for selected years, demonstrating agreements between STEM-LAI 250, STEM-LAI $250 *$ (without consistency adjustment of LAI inputs), linearly interpolated LAI ( $\left.\mathrm{LND}_{\text {lin }}\right)$, and in-situ measured LAI.

483 Fig. 9a showcases the degree of manipulation of the MODIS $\mathrm{LAI}_{250}$ as a result of the consistency 484 adjustment for a Landsat overpass date (Sep. 1, 2010). While the overall spatial patterns are similar in 485 the two $\mathrm{LAI}_{250}$ versions, the original $\mathrm{LAI}_{250}$ has a smoother appearance and displays obvious differences 486 from the Landsat based LAI retrievals in several places. The consistency adjustment is critical for 487 ensuring that the medium resolution data provide correct temporal information for the high spatial 488 resolution data and for producing a realistic temporal behavior of the 4-day $30 \mathrm{~m}$ resolution LAI 489 between Landsat acquisitions (Fig. 9b). Abrupt and unrealistic LAI fluctuations sometimes occur when 490 the original MODIS LAI 250 is used as input to STEM-LAI (Fig. 9b). While the use of an $\mathrm{LAI}_{30}$ time491 series generated by linearly interpolating LAI retrievals between Landsat acquisitions (LND lin) appear to 
Table 2 Quantitative statistic measures of the performance of STEM-LAI, based on MODIS 250 and $1 \mathrm{~km}$ LAI products, in estimating LAI $\left[\mathrm{m}^{2} \mathrm{~m}^{-2}\right]$ over maize and soybean. Also shown are the performances achieved by linearly interpolating LAI retrievals between Landsat scenes $\left(\mathrm{LND}_{250 \text {, lin }}\right.$ and $\left.\mathrm{LND}_{1 \mathrm{~km} \text {, lin }}\right)$. The LAI retrievals were evaluated against in-situ measurements ( $\mathrm{n}=257$ and 99 for maize and soybean, respectively) collected over 11 growing seasons $(2002-2012)$.

$\mathrm{O}$ is the mean of the in-situ measurements, $\mathrm{S}$ is the mean of the retrievals, RMSD is the rootmean-square difference between model estimates and measurements, rRMSD is the relative RMSD (RMSD/O*100), MBE is the mean bias error (if positive measurements are being overestimated), $\mathrm{r}^{2}$ is the coefficient of determination, and $\mathrm{E}$ is the coefficient of efficiency.

\begin{tabular}{|c|c|c|c|c|c|c|c|c|}
\hline & & $O$ & $S$ & $R M S D$ & $r R M S D$ & $M B E$ & $r^{2}$ & $E$ \\
\hline \multirow{2}{*}{$\begin{array}{l}\text { STEM- } \\
\text { LAI I }_{250}\end{array}$} & Corn & 3.00 & 2.22 & 1.21 & 40.4 & -0.77 & 0.86 & 0.65 \\
\hline & Soyb & 1.84 & 1.78 & 0.57 & 31.1 & -0.07 & 0.86 & 0.87 \\
\hline \multirow{2}{*}{$\mathbf{L N D}_{250, \text { iin }}$} & Corn & 3.00 & 2.30 & 1.29 & 42.8 & -0.70 & 0.72 & 0.61 \\
\hline & Soyb & 1.84 & 1.76 & 0.65 & 35.4 & -0.08 & 0.81 & 0.84 \\
\hline \multirow{2}{*}{$\begin{array}{l}\text { STEM- } \\
\text { LAI }_{1 \mathrm{~km}}\end{array}$} & Corn & 3.00 & 2.27 & 1.26 & 41.9 & -0.73 & 0.78 & 0.62 \\
\hline & Soyb & 1.84 & 1.98 & 0.62 & 33.7 & 0.13 & 0.80 & 0.85 \\
\hline \multirow{2}{*}{$\mathbf{L N D}_{1 \mathrm{~km}, \mathrm{lin}}$} & Corn & 3.00 & 2.27 & 1.26 & 41.9 & -0.73 & 0.67 & 0.60 \\
\hline & Soyb & 3.06 & 1.89 & 0.60 & 32.7 & 0.05 & 0.81 & 0.86 \\
\hline
\end{tabular}

492 provide a reasonable approximation to the measured LAI time-series, this approach can be problematic 493 during periods of rapid vegetation growth or when Landsat acquisitions are far apart (Fig. 9b).

As indicated in Fig. 9b, STEM-LAI retrievals are biased low over the maize fields, which are also corroborated by the full evaluation of STEM-LAI retrievals against the 11-year record of in-situ measurements (Fig. 10a and Table 2). While significantly correlated, retrievals based on both MODIS $\mathrm{LAI}_{250}$ (Fig. 10a) and $\mathrm{LAI}_{1 \mathrm{~km}}$ (Fig. 10b) underestimate the destructively measured maize LAI by $31 \%$ (linear regression slope $=0.69)$. A similar tendency was reported by Houborg et al. (2015b) over the same sites, but based on retrievals from a regularized model inversion system, which were attributed in part to significant foliage clumping not being properly accounted for by the 1-D radiative transfer model. Foliage clumping in maize canopies has been reported in several studies (Demarez et al., 2008;

503 Duthoit et al., 2008; España et al., 1998; López-Lozano et al., 2007) and the regular distribution of 504 leaves (i.e. a clumping factor that is larger than unity) assumed in the 3-dimensional radiative transfer 505 model invoked for broadleaf crops in the MODIS LAI algorithm (Knyazikhin et al., 1999) may provide 506 an explanation for the biased LAI estimates. 
a)
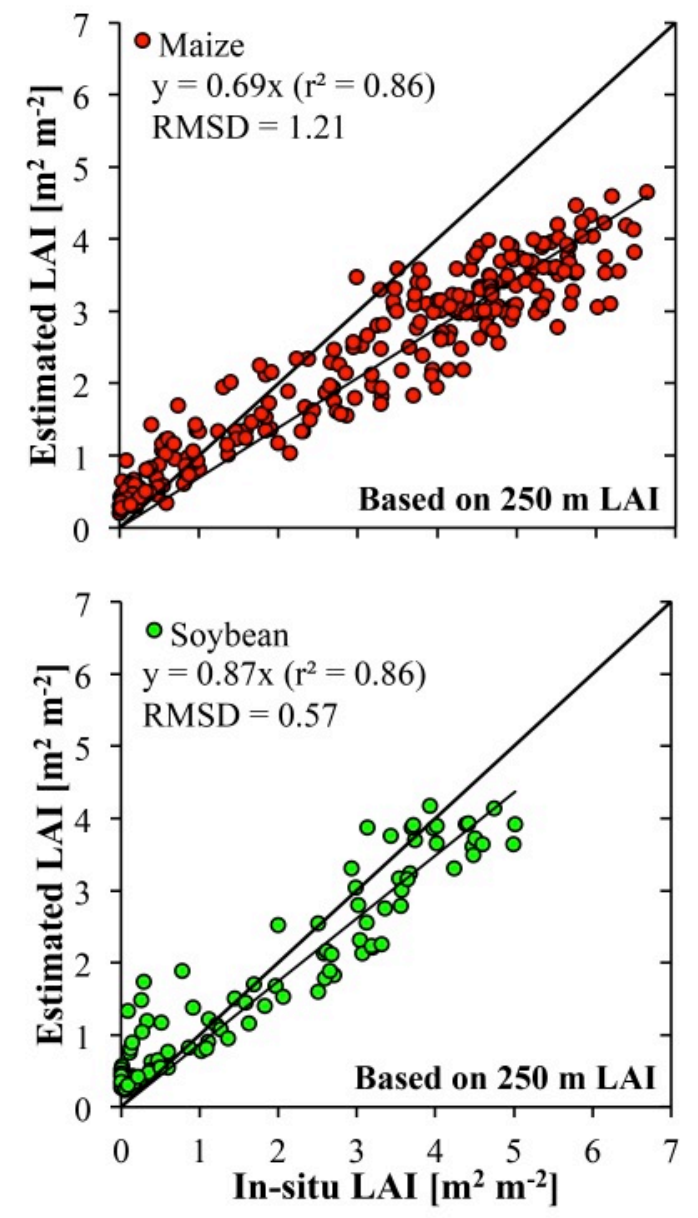

b)
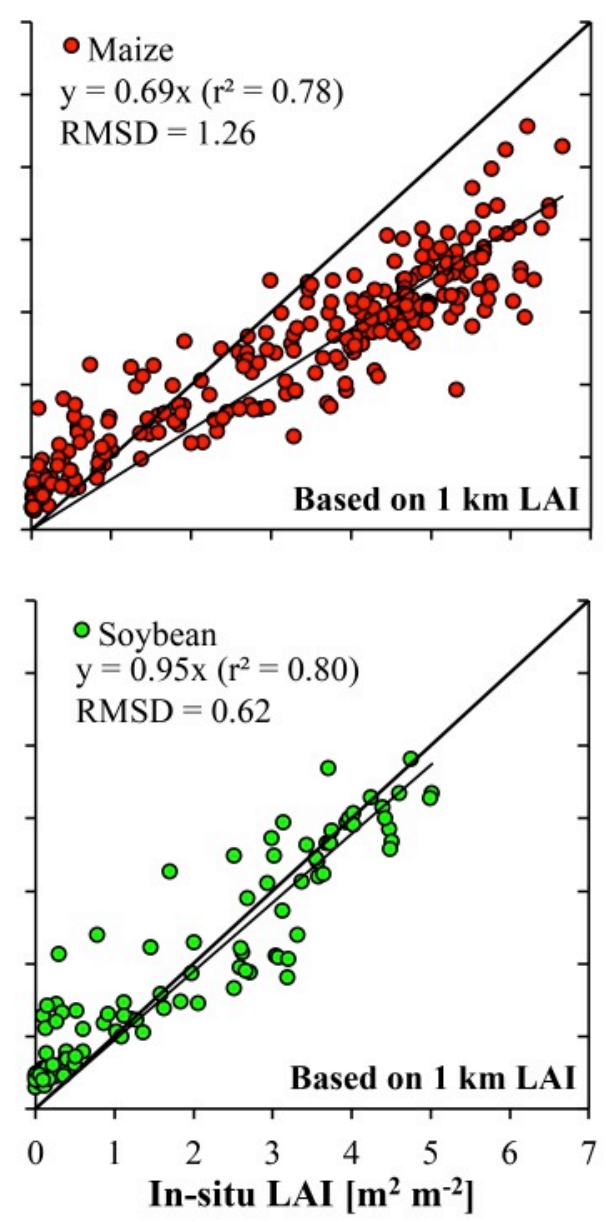

Fig. 10 LAI scatter plots comparing in-situ measured LAI in maize and soybean against LAI estimated using STEM-LAI 250 (a) and STEM-LAI $1 \mathrm{~km}$ (b). Full statistic metrics are reported in Table 2.

In both maize and soybean, the use of $\mathrm{LAI}_{250}$ rather than $\mathrm{LAI}_{1 \mathrm{~km}}$ as input to STEM-LAI leads to improvements in the agreement with the measured LAI (Fig. 10, Table 2). STEM-LAI 250 also outperforms LAI retrievals generated through linear interpolation of Landsat LAI between overpasses $\left(\mathrm{LND}_{\text {lin }}\right)($ Table 2$)$. In all cases, reduced agreement is likely to occur during periods of rapid vegetation growth or decay, where the MODIS 8-day composited estimates may not provide a good approximation of day-specific LAI dynamics (Gao et al., 2012).

As a further test of the utility of STEM-LAI for describing the temporal behavior of LAI between Landsat acquisitions, Fig. 11 compares the performance (i.e. RMSD) of STEM-LAI ${ }_{250}$ versus LND $_{\text {lin. }}$. The RMSD between satellite estimates and in-situ measurements is reported as a function of the number of days from a Landsat overpass, calculated based on available samples (n) within 6-day intervals over the 11 study years. When the measurement date is relatively close to a Landsat acquisition, LND $_{\text {lin }}$ 


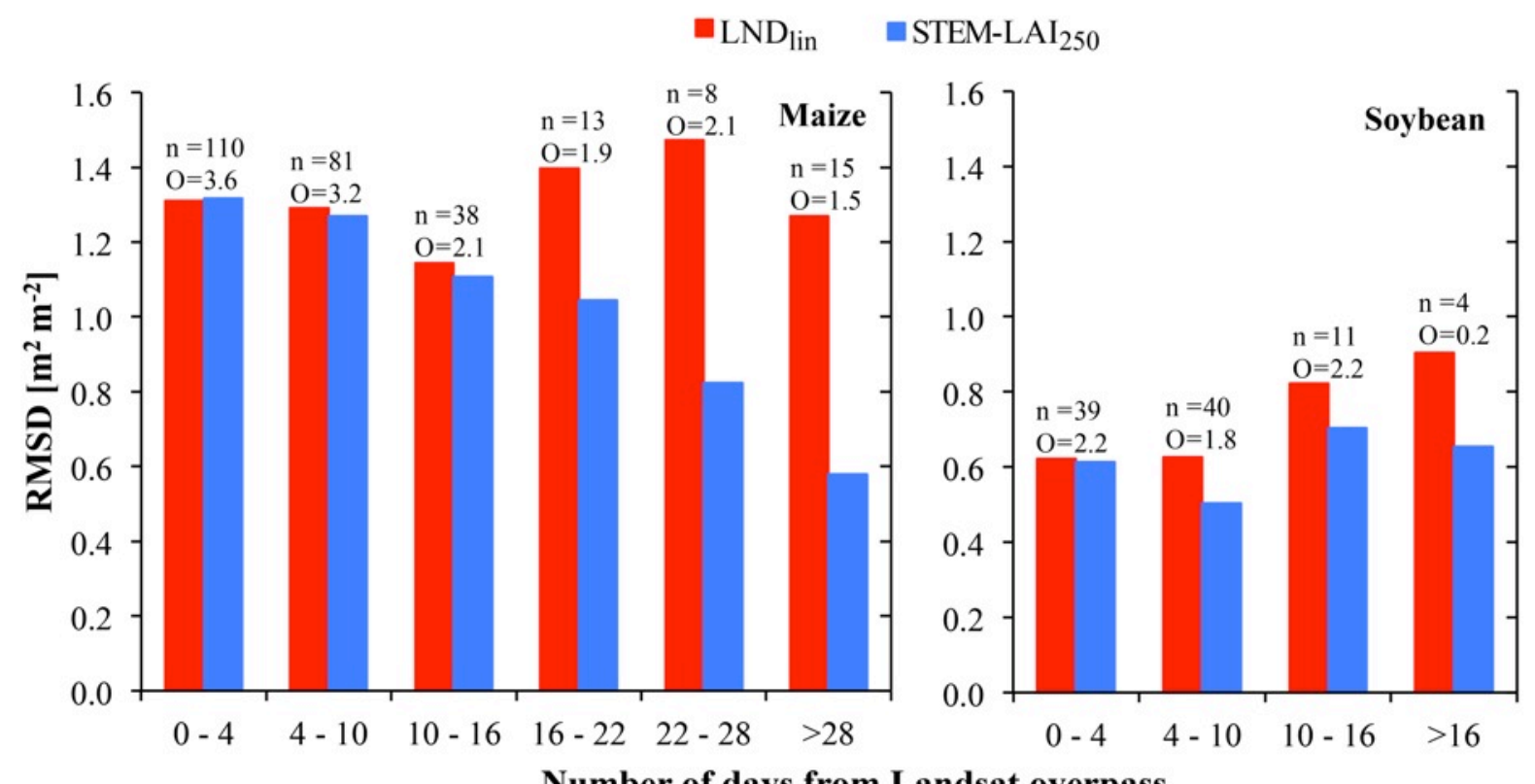

Fig. 11 Utility of STEM-LAI as a mechanism for interpolating LAI between Landsat acquisitions, demonstrated by comparing prediction accuracies (i.e. RMSD) of STEM-LAI 250 and LND $_{\text {lin }}$ (4-day LAI estimated by linearly interpolating between Landsat acquisition) as a function of the number of days to nearest Landsat overpass. The number of samples (n) and LAI measurement mean $(\mathrm{O})$ within each 6-day interval are provided above the bars.

520 provides a good approximation, although STEM-LAI 250 performs better in all cases. Beyond 16 days, 521 the use of STEM-LAI 250 predictions show a substantial improvement over LND $_{\text {lin }}$ with up to an 522 approximately 50\% reduction in RMSD (Fig. 11). These results demonstrate the utility of STEM-LAI as 523 a powerful and effective mechanism for interpolating spatio-temporal features in LAI between Landsat 524 acquisitions.

\subsection{Opportunities and challenges in applications of STEM-LAI}

528 In this study, STEM-LAI was applied to an agricultural region in Nebraska, producing 4-day time-series 529 of $30 \mathrm{~m}$ resolution LAI over a period of 11 years (2002-2012) using MODIS LAI and available 530 Landsat-5 and Landsat-7 scenes as input. A completely automated processing stream has been 531 developed that only requires the specification of a region of interest including scene dimensions and 532 center geolocation. An online submission system is being planned for accepting web-based processing requests that will be queued on a central in-house processing unit before automatic execution and 
534 product delivery. Initially, requests will be limited to smaller subsets over a range of test sites. Larger535 scale implementations will be feasible once the code has been optimized for parallel processing. In 536 principle, STEM-LAI can be applied to any region and time period with an availability of MODIS LAI 537 and Landsat imagery. It is not restricted to agricultural land cover types and is able to spatially and 538 temporally disaggregate MODIS medium resolution LAI irrespective of biome specification. Further 539 assessing actual performances of the STEM-LAI approach over contrasting environments and 540 landscapes based on expansive ground-based datasets remains an important objective for future 541 research.

543 While regions with extensive cloud cover will result in a more limited number of usable Landsat scenes, 544 high predictabilities of STEM-LAI were recorded in this study even with extended periods (2-4 months) 545 between the Landsat pair and prediction date (Table 1 and Fig. 7). In fact, STEM-LAI may be 546 particularly beneficial in environments characterized by only a few good Landsat acquisitions over the 547 growing season, where a linear interpolation of LAI between acquisitions will be associated with 548 substantial uncertainties (Fig. 11). STARFM was setup to use MODIS-Landsat pairs from one 549 acquisition date at a time, with selections determined via the maximum correlation of MODIS LAI 550 between pair and prediction date. A consequence of this is that if a selected Landsat scene is cloud 551 contaminated or contains SLC-off stripes, gaps will exist in the corresponding locations for the 552 prediction image. For efficient gap-filling purposes, STARFM was modified to iterate through a 553 sequence of plausible pair dates until all gaps in the prediction images have been filled. Such an 554 approach produced prediction imagery with realistic spatial patterns in Landsat imagery affected by 555 striping (Fig. 7c) and facilitated spatially complete (no gaps) $\mathrm{LAI}_{30}$ maps over the full duration of the 556 study period. While there are a range of gap-filling approaches that might prove useful (Mariethoz et al., 557 2012), a comprehensive evaluation of the utility of STEM-LAI gap-filling of clouds and SLC-off stripes 558 in Landsat imagery is needed.

560 A focus of the current analysis was to define optimal STARFM parameters (i.e. $w$ and $s$ ) for the study 561 region. While the adopted mechanisms for informing the selection of similar neighboring pixels (i.e. 562 land cover classification versus internal threshold method) is directly adaptable to other environments 563 and study regions, the parameterization of $w$ and $s$ may need modification to reflect the heterogeneity 564 and complexity of any particular study region (Gao et al., 2006). A routine is currently being developed 
565 to automatically derive optimal parameter values for a given study area based on STARFM runs for a

566 few selected cases, with varying duration between pair and prediction date and subsequent verification 567 against actual Landsat LAI (as demonstrated in Table 1).

569 The availability of a sufficient number of homogenous medium resolution LAI samples for the various 570 land cover types within the study region is a key requirement for reliable downscaling of MODIS LAI to 571 Landsat scale using the regression tree approach (Section 3.1.1). At the same time, medium resolution 572 homogeneous pixels are assumed to provide identical temporal changes as high resolution observations 573 from the same class (Gao et al., 2012). However, these conditions can be difficult to satisfy with input of $574 \mathrm{LAI}_{1 \mathrm{~km}}$, particularly in heterogeneous agricultural landscapes. In this study large improvements were 575 observed by using input of $\mathrm{LAI}_{250}$, downscaled from the $1 \mathrm{~km}$ MODIS products, based on a scale576 consistent NDVI driven biome-specific approach. $\mathrm{LAI}_{250}$ provides a better representation of land cover 577 specific features in Landsat imagery, with a substantial increase in the number of homogeneous training 578 samples. Such an approach provides a better foundation for acquiring a sufficient number of LAI 579 references, particularly over dense vegetation, to avoid underestimation for high LAI (Gao et al., 2014). 580 The implementation of $\mathrm{LAI}_{250}$ is expected to be especially critical for STEM-LAI applications in 581 environments with a complex mixture of land cover types, or in places with relatively few vegetated 582 elements, such as desert environments with isolated areas of irrigated agriculture, where the collection of 583 sufficient homogeneous samples may be challenging.

585 The fidelity of the downscaled $\mathrm{LAI}_{250}$ estimates depends on the validity of the established biome586 specific LAI-NDVI relationships (Section 3.1.1). However, since consistency with the $1 \mathrm{~km}$ estimates is 587 ensured, only the relative sub-pixel variability from the $1 \mathrm{~km}$ LAI value is affected. Presumably, the 588 definition of model-diagnosed LAI-NDVI relationships with a better account of spatio-temporal 589 variations in soil background, leaf and canopy biochemical and structural characteristics, atmospheric 590 conditions, and view and illumination geometry (Houborg et al., 2015b), would improve $1 \mathrm{~km}$ to $250 \mathrm{~m}$ 591 downscaling accuracies further. Alternatively, rule-based multivariate linear regressions for $\mathrm{LAI}_{250}$ 592 estimation may be constructed based on MODIS LAI $1 \mathrm{~km}$ and red and near-infrared band reflectances at $593250 \mathrm{~m}$ resolution, using the same regression tree approach adopted for Landsat data, which is viable 594 given the importance of these two bands in LAI retrievals (Gao et al., 2012). For downscaling to 595 Landsat-scale LAI, the cubist-based regression trees produced robust linear regression models with 
596 varying contributions from the input Landsat spectral bands, typically with average errors below 0.2 and 597 correlation coefficients above 0.92 .

STEM-LAI was demonstrated using the 8-day combined Terra and Aqua MODIS LAI product, but it can be extended to other available global medium resolution LAI products. A parallel research effort focuses on the integration of multi-product $1 \mathrm{~km}$ resolution LAI data streams to facilitate efficient product validation and intercomparison over defined regions of interest. The additional products currently include 1) the 10-day GEOV1 LAI product, which represents a fusion of CYCLOPES and MODIS products produced at $1 / 112^{\circ}$ resolution between 2001-2014 as part of the Copernicus Global Land Surface (Baret et al., 2013), and 2) the 8-day Global Land Surface Satellite (GLASS) LAI product integrating both MODIS and CYCLOPES LAI within a neural network approach (Xiao et al., 2013) and available between 2001-2012. An expected outcome of this research is a combined temporally continuous (4-day) and spatially enhanced $(30 \mathrm{~m})$ product with assigned uncertainties based on the spread in estimates between the adopted suite of global products. This could serve as a well-constrained

\section{Conclusions}

An application (STEM-LAI) has been developed for automated downscaling of medium resolution LAI products to achieve both high spatial $(30 \mathrm{~m})$ and temporal (4-day) resolution for regions of interest. STEM-LAI utilizes a regression tree approach for establishing predictive relationships between a reference LAI dataset (e.g., MODIS) and pixel-aggregated Landsat surface reflectances, which are then applied to Landsat imagery for estimating LAI at $30 \mathrm{~m}$ resolution during Landsat acquisition dates. A data fusion algorithm (STARFM) is then applied to scale-consistent LAI data streams of relatively high frequency or high spatial resolution to effectively interpolate high spatial resolution LAI information between Landsat acquisitions. Application to the MODIS LAI product demonstrates the utility of STEM-LAI for reproducing spatial features and magnitudes observed in actual Landsat LAI images for a wide range of plant development stages and times between coincident (MODIS-Landsat) overpass and prediction dates. However, for the highly heterogeneous agricultural region studied here, LAI at $250 \mathrm{~m}$ resolution (downscaled from MODIS $1 \mathrm{~km} \mathrm{LAI)} \mathrm{was} \mathrm{required} \mathrm{to} \mathrm{achieve} \mathrm{high} \mathrm{fidelity} \mathrm{results,} \mathrm{due} \mathrm{to}$ limitations in the availability of homogeneous pixels at the $1 \mathrm{~km}$ scale. 
628 Refinements to the implementation and execution of STARFM were implemented, including: 1) 629 techniques for ensuring precise geolocation and consistency between input LAI data streams, 2) a 630 sequential MODIS-Landsat pair selection approach for effective gap-filling of areas affected by clouds 631 or Landsat-7 SLC-off stripes, and 3) optimized definitions of tunable parameters and input options. 632 These modifications were effective at reliably describing the fine-scale temporal behavior in LAI 633 between Landsat acquisitions, while simultaneously filling gaps in Landsat imagery.

635 Evaluation against an 11-year record of in-situ destructively sampled LAI over maize and soybean 636 showed that STEM-LAI was able to accurately reproduce the observed dynamics $\left(\mathrm{r}^{2}=0.86\right.$ for both 637 crops), although estimates were biased low over maize (31\% underestimation). The low bias was partly 638 attributed to significant foliage clumping that conflicts with the leaf distribution (regular) assumed for 639 broadleaf crops in the MODIS LAI algorithm. Compared to linear interpolation of LAI time-series 640 between acquisitions, STEM-LAI was superior in predicting the observed LAI when the in-situ 641 measurement date was more than 10 days away from the nearest Landsat acquisition, with up to 50\% 642 reductions in prediction error.

644 STEM-LAI provides a novel interface for the downscaling of medium resolution LAI, which can be readily adapted to other environments and landscapes with variations in cloud cover conditions, land 646 cover types, and surface heterogeneity. STEM-LAI also has the capacity to be extended to other 647 available global LAI products. Such an extension would help to establish robust temporally continuous 648 and spatially enhanced estimates of LAI with assigned uncertainties based on the spread between the 649 adopted suite of global products. STEM-LAI enhances the information content of existing retrievals, 650 which can be beneficial to a wide range of application fields requiring time-critical information on 651 vegetation density at sub-field scales.

\section{6. Acknowledgements}

655 The research undertaken here was funded by the King Abdullah University of Science and Technology 656 (KAUST). We greatly appreciate the biological datasets provided by the Center for Advanced Land 
Management Information Technologies (CALMIT) and the Carbon Sequestration Program, University

of Nebraska-Lincoln.

\section{References}

Anderson, M.C., Kustas, W.P., Alfieri, J.G., Gao, F., Hain, C., Prueger, J.H., Evett, S., Colaizzi, P., Howell, T., Chávez, J.L., 2012. Mapping daily evapotranspiration at Landsat spatial scales during the BEAREX'08 field campaign. Adv. Water Resour. 50, 162-177. doi:10.1016/j.advwatres.2012.06.005

Anderson, M.C., Zolin, C., Hain, C.R., Semmens, K., Tugrul Yilmaz, M., Gao, F., 2015. Comparison of satellite-derived LAI and precipitation anomalies over Brazil with a thermal infrared-based Evaporative Stress Index for 2003-2013. J. Hydrol. 526, 287-302. doi:10.1016/j.jhydrol.2015.01.005

Baret, F., Hagolle, O., Geiger, B., Bicheron, P., Miras, B., Huc, M., Berthelot, B., Niño, F., Weiss, M., Samain, O., Roujean, J.L., Leroy, M., 2007. LAI, fAPAR and fCover CYCLOPES global products derived from VEGETATION. Remote Sens. Environ. 110, 275-286. doi:10.1016/j.rse.2007.02.018

Baret, F., Weiss, M., Lacaze, R., Camacho, F., Makhmara, H., Pacholcyzk, P., Smets, B., 2013. GEOV1: LAI and FAPAR essential climate variables and FCOVER global time series capitalizing over existing products. Part1: Principles of development and production. Remote Sens. Environ. 137, 299-309. doi:10.1016/j.rse.2012.12.027

Bonan, G.B., Lawrence, P.J., Oleson, K.W., Levis, S., Jung, M., Reichstein, M., Lawrence, D.M., Swenson, S.C., 2011. Improving canopy processes in the Community Land Model version 4 (CLM4) using global flux fields empirically inferred from FLUXNET data. J. Geophys. Res. 116, 1-22. doi:10.1029/2010JG001593

Cai, S., Liu, D., Sulla-Menashe, D., Friedl, M.A., 2014. Enhancing MODIS land cover product with a spatial-temporal modeling algorithm. Remote Sens. Environ. 147, 243-255. doi:10.1016/j.rse.2014.03.012

Cammalleri, C., Anderson, M.C., Gao, F., Hain, C.R., Kustas, W.P., 2014. Mapping daily evapotranspiration at field scales over rainfed and irrigated agricultural areas using remote sensing data fusion. Agric. For. Meteorol. 186, 1-11. doi:10.1016/j.agrformet.2013.11.001

Demarez, V., Duthoit, S., Baret, F., Weiss, M., Dedieu, G., 2008. Estimation of leaf area and clumping indexes of crops with hemispherical photographs. Agric. For. Meteorol. 148, 644-655. doi:10.1016/j.agrformet.2007.11.015

Doraiswamy, P., Hatfield, J., Jackson, T., Akhmedov, B., Prueger, J., Stern, A., 2004. Crop condition and yield simulations using Landsat and MODIS. Remote Sens. Environ. 92, 548-559. doi:10.1016/j.rse.2004.05.017

Duthoit, S., Demarez, V., Gastellu-Etchegorry, J.-P., Martin, E., Roujean, J.-L., 2008. Assessing the effects of the clumping phenomenon on BRDF of a maize crop based on 3D numerical scenes using DART model. Agric. For. Meteorol. 148, 1341-1352. doi:10.1016/j.agrformet.2008.03.011

Emelyanova, I. V., McVicar, T.R., Van Niel, T.G., Li, L.T., van Dijk, A.I.J.M., 2013. Assessing the accuracy of blending Landsat-MODIS surface reflectances in two landscapes with contrasting spatial and temporal dynamics: A framework for algorithm selection. Remote Sens. Environ. 133, 193-209. doi:10.1016/j.rse.2013.02.007 
España, M., Baret, F., Chelle, M., Aries, F., Andrieu, B., 1998. A dynamic model of maize 3D architecture: Application to the parameterisation of the clumpiness of the canopy. Agronomie 18, 609-626.

French, A., 2001. Scaling of surface energy fluxes using remotely sensed data. University of Maryland. Ganguly, S., Nemani, R.R., Zhang, G., Hashimoto, H., Milesi, C., Michaelis, A., Wang, W., Votava, P., Samanta, A., Melton, F., Dungan, J.L., Vermote, E., Gao, F., Knyazikhin, Y., Myneni, R.B., 2012. Generating global Leaf Area Index from Landsat: Algorithm formulation and demonstration. Remote Sens. Environ. 122, 185-202. doi:10.1016/j.rse.2011.10.032

Gao, F., Anderson, M.C., Kustas, W.P., Houborg, R., 2014. Retrieving Leaf Area Index From Landsat Using MODIS LAI Products and Field Measurements. IEEE Geosci. Remote Sens. Lett. 11, 773777. doi:10.1109/LGRS.2013.2278782

Gao, F., Anderson, M.C., Kustas, W.P., Wang, Y., 2012. Simple method for retrieving leaf area index from Landsat using MODIS leaf area index products as reference. J. Appl. Remote Sens. 6, 6351563554. doi:10.1117/1.JRS.6.063554

Gao, F., Masek, J., Schwaller, M., Hall, F., 2006. On the blending of the Landsat and MODIS surface reflectance: predicting daily Landsat surface reflectance. IEEE Trans. Geosci. Remote Sens. 44, 2207-2218. doi:10.1109/TGRS.2006.872081

Gitelson, A.A., Peng, Y., Arkebauer, T.J., Schepers, J., 2014. Relationships between gross primary production, green LAI, and canopy chlorophyll content in maize: Implications for remote sensing of primary production. Remote Sens. Environ. 144, 65-72. doi:10.1016/j.rse.2014.01.004

Gitelson, A.A., Vina, A., Arkebauer, T.J., Rundquist, D.C., Keydan, G., Leavitt, B., 2003. Remote estimation of leaf area index and green leaf biomass in maize canopies. Geophys. Res. Lett. 30, 1248. doi:10.1029/2002GL016450

Gobron, N., Pinty, B., Verstraete, M.M., 1997. Theoretical limits to the estimation of the leaf area index on the basis of visible and near-infrared remote sensing data. IEEE Trans. Geosci. Remote Sens. 35, 1438-1445. doi: $10.1109 / 36.649798$

Hilker, T., Wulder, M. a., Coops, N.C., Seitz, N., White, J.C., Gao, F., Masek, J.G., Stenhouse, G., 2009. Generation of dense time series synthetic Landsat data through data blending with MODIS using a spatial and temporal adaptive reflectance fusion model. Remote Sens. Environ. 113, 1988-1999. doi:10.1016/j.rse.2009.05.011

Houborg, R., Fisher, J., Skidmore, A., 2015a. Advances in remote sensing of vegetation function and traits. Int. J. Appl. Earth Obs. Geoinf. doi:10.1016/j.jag.2015.06.001

Houborg, R., McCabe, M., Cescatti, A., Gao, F., Schull, M., Gitelson, A., 2015b. Joint leaf chlorophyll content and leaf area index retrieval from Landsat data using a regularized model inversion system (REGFLEC). Remote Sens. Environ. 159, 203-221. doi:10.1016/j.rse.2014.12.008

Huang, D., Knyazikhin, Y., Wang, W., Deering, D., Stenberg, P., Shabanov, N., Tan, B., Myneni, R., 2008. Stochastic transport theory for investigating the three-dimensional canopy structure from space measurements. Remote Sens. Environ. 112, 35-50. doi:10.1016/j.rse.2006.05.026

Knyazikhin, Y. et al, 1999. MODIS Leaf Area Index (LAI) and Fraction of Photosynthetically Active Radiation Absorbed by Vegetation (FPAR) Product (MOD15) Algorithm Theoretical Basis Document.

Legates, D.R., McCabe, G.J., 1999. Evaluating the use of "goodness-of-fit" Measures in hydrologic and hydroclimatic model validation. Water Resour. Res. 35, 233-241. doi:10.1029/1998WR900018

López-Lozano, R., Baret, F., Chelle, M., Rochdi, N., España, M., 2007. Sensitivity of gap fraction to maize architectural characteristics based on 4D model simulations. Agric. For. Meteorol. 143, $217-$ 229. doi:10.1016/j.agrformet.2006.12.005 
Mariethoz, G., McCabe, M.F., Renard, P., 2012. Spatiotemporal reconstruction of gaps in multivariate fields using the direct sampling approach. Water Resour. Res. 48, 1-13. doi: $10.1029 / 2012$ WR 012115

Maxwell, S.K., Schmidt, G.L., Storey, J.C., 2007. A multi scale segmentation approach to filling gaps in Landsat ETM+ SLC off images. Int. J. Remote Sens. 28, 5339-5356. doi:10.1080/01431160601034902

Moran, M.S., Inoue, Y., Barnes, E.M., 1997. Opportunities and limitations for image-based remote sensing in precision crop management. Remote Sens. Environ. 61, 319-346. doi:10.1016/S00344257(97)00045-X

Myneni, R.B., Ramakrishna, R., Nemani, R., Running, S.W., 1997. Estimation of global leaf area index and absorbed par using radiative transfer models. IEEE Trans. Geosci. Remote Sens. 35, 13801393. doi:10.1109/36.649788

Pinty, B., Jung, M., Kaminski, T., Lavergne, T., Mund, M., Plummer, S., Thomas, E., Widlowski, J.-L., 2011. Evaluation of the JRC-TIP $0.01^{\circ}$ products over a mid-latitude deciduous forest site. Remote Sens. Environ. 115, 3567-3581. doi:10.1016/j.rse.2011.08.018

Roy, D.P., Ju, J., Lewis, P., Schaaf, C., Gao, F., Hansen, M., Lindquist, E., 2008. Multi-temporal MODIS-Landsat data fusion for relative radiometric normalization, gap filling, and prediction of Landsat data. Remote Sens. Environ. 112, 3112-3130. doi:10.1016/j.rse.2008.03.009

Schmidt, G.L., Jenkerson, C.B., Masek, J., Vermote, E., Gao, F., 2013. Landsat Ecosystem Disturbance Adaptive Processing System (LEDAPS) Algorithm Description. U.S. Geol. Surv. Open-File Rep. 2013-1057 $17 \mathrm{p}$.

Sellers, P., Randall, D., Collatz, G., Berry, J., Field, C., Dazlich, D., Zhang, C., Collelo, G., Bounoua, L., 1996. A revised land surface parameterization (SiB2) for atmospheric GCMs. Part I: Model Formulation. J. Clim. 9, 676-705.

Senf, C., Leitão, P.J., Pflugmacher, D., van der Linden, S., Hostert, P., 2015. Mapping land cover in complex Mediterranean landscapes using Landsat: Improved classification accuracies from integrating multi-seasonal and synthetic imagery. Remote Sens. Environ. 156, 527-536. doi:10.1016/j.rse.2014.10.018

Verma, S.B., Dobermann, A., Cassman, K.G., Walters, D.T., Knops, J.M., Arkebauer, T.J., Suyker, A.E., Burba, G.G., Amos, B., Yang, H., Ginting, D., Hubbard, K.G., Gitelson, A. a., Walter-Shea, E. a., 2005. Annual carbon dioxide exchange in irrigated and rainfed maize-based agroecosystems. Agric. For. Meteorol. 131, 77-96. doi:10.1016/j.agrformet.2005.05.003

Walker, J.J., de Beurs, K.M., Wynne, R.H., 2014. Dryland vegetation phenology across an elevation gradient in Arizona, USA, investigated with fused MODIS and Landsat data. Remote Sens. Environ. 144, 85-97. doi:10.1016/j.rse.2014.01.007

Wang, P., Gao, F., Masek, J.G., 2014. Operational Data Fusion Framework for Building Frequent Landsat-Like Imagery. IEEE Trans. Geosci. Remote Sens. 52, 7353-7365. doi:10.1109/TGRS.2014.2311445

Weng, Q., Fu, P., Gao, F., 2014. Generating daily land surface temperature at Landsat resolution by fusing Landsat and MODIS data. Remote Sens. Environ. 145, 55-67. doi:10.1016/j.rse.2014.02.003

Xiao, Z., Liang, S., Wang, J., Chen, P., Yin, X., Zhang, L., Song, J., 2013. Use of General Regression Neural Networks for Generating the GLASS Leaf Area Index Product. IEEE Trans. Geosci. Remote Sens. 52, 1-15.

Zhang, X., Friedl, M.A., Schaaf, C.B., 2006. Global vegetation phenology from Moderate Resolution Imaging Spectroradiometer (MODIS): Evaluation of global patterns and comparison with in situ measurements. J. Geophys. Res. Biogeosciences 111, 1-14. doi:10.1029/2006JG000217 
792 Zhu, Z., Woodcock, C.E., 2012. Object-based cloud and cloud shadow detection in Landsat imagery.

793 Remote Sens. Environ. 118, 83-94. doi:10.1016/j.rse.2011.10.028

794 